TRANSACTIONS OF THE

AMERICAN MATHEMATICAL SOCIETY

Volume 356, Number 12, Pages 4701-4736

S 0002-9947(04)03663-3

Article electronically published on June 22, 2004

\title{
QUATERNIONIC ALGEBRAIC CYCLES AND REALITY
}

\author{
PEDRO F. DOS SANTOS AND PAULO LIMA-FILHO
}

\begin{abstract}
In this paper we compute the equivariant homotopy type of spaces of algebraic cycles on real Brauer-Severi varieties, under the action of the Galois group $\operatorname{Gal}(\mathbb{C} / \mathbb{R})$. Appropriate stabilizations of these spaces yield two equivariant spectra. The first one classifies Dupont/Seymour's quaternionic $K$ theory, and the other one classifies an equivariant cohomology theory $\mathfrak{Z}^{*}(-)$ which is a natural recipient of characteristic classes $K H^{*}(X) \rightarrow \mathfrak{Z}^{*}(X)$ for quaternionic bundles over Real spaces $X$.
\end{abstract}

\section{INTRODUCTION}

In Ati66 Atiyah developed a $K$-theory for spaces $(X, \sigma)$ with an involution $\sigma$, the Real spaces in his terminology. The construction uses the notion of a Real bundle $(E, \tau)$ over $(X, \sigma)$ which consists of a complex vector bundle $E$ over $X$, along with an anti-linear map $\tau: E \rightarrow E$ covering the involution $\sigma$ and satisfying $\tau^{2}=1$. The group $K R(X)$ is then defined as the Grothendieck group of the monoid of isomorphism classes of Real bundles over $(X, \sigma)$, and the resulting theory is called $K R$-theory.

In a similar fashion, J. Dupont developed in Dup69 the symplectic $K$-theory $K S p(X)$ for Real spaces $(X, \sigma)$. His construction is similar to Atiyah's, in that $K S p(X)$ is the Grothendieck group of the monoid of isomorphism classes of symplectic bundles over $(X, \sigma)$. In this context, a symplectic bundle $(E, \tau)$ over $(X, \sigma)$ consists of a complex vector bundle $E$ over $X$, along with an anti-linear map $\tau: E \rightarrow E$ covering the involution $\sigma$ and satisfying $\tau^{2}=-1$. Subsequently, R. M. Seymour reintroduced this theory in Sey73], where he called it quaternionic K-theory and denoted it by $K H(X)$. We adopt this terminology, for it avoids confusion with the non-equivariant notion of symplectic $K$-theory.

A clear and conceptual reason for the existence of these two competing theories arises when one tries to find their respective classifying spaces in the equivariant category. In fact, one can extend these theories to $R O(\mathbb{Z} / 2)$-graded cohomology theories $K R^{*}$ and $K H^{*}$ in the sense of Seg68 and May77]. To this purpose, one constructs $\mathbb{Z} / 2$-spaces $(\mathbb{Z} \times B U)_{\mathbb{R}}$ and $(\mathbb{Z} \times B U)_{\mathbb{H}}$ satisfying

$$
\left[X_{+},(\mathbb{Z} \times B U)_{\mathbb{R}}\right]_{\mathbb{Z} / 2} \cong K R(X) \text { and }\left[X_{+},(\mathbb{Z} \times B U)_{\mathbb{H}}\right]_{\mathbb{Z} / 2} \cong K H(X) ;
$$

cf. LLFM98b and Proposition 4.3, respectively. These spaces are shown to have the structure of $\mathbb{Z} / 2$-equivariant infinite loop spaces in [LLFM98b] and Theorem

Received by the editors October 9, 2001.

2000 Mathematics Subject Classification. Primary 55P91; Secondary 19L47, 14 C25.

The first author was supported in part by FCT (Portugal) through program POCTI and grant POCTI/1999/MAT/34015. The second author was partially supported by the NSF. 
4.6, respectively, yielding the spectra classifying the desired equivariant cohomology theories.

In order to construct such classifying spaces, we first identify $\mathbb{Z} / 2$ - the underlying group of the equivariant category - with the Galois group $\operatorname{Gal}(\mathbb{C} / \mathbb{R})$. Recall that the Brauer group [Gro57] $B r(\mathbb{R})$ of $\mathbb{R}$ is also isomorphic to $\mathbb{Z} / 2$. This will be shown to account for the two distinct $K$-theories in Section 4 The argument is roughly the following. Let $\mathbb{P}\left(\mathbb{C}^{n}\right)$ denote the projective space of complex 1-dimensional subspaces of $\mathbb{C}^{n}$. In the language of schemes, this is the set of complex-valued points of $\mathbb{P}^{n-1}$, endowed with the analytic topology. The Galois group $\mathbb{Z} / 2=\operatorname{Gal}(\mathbb{C} / \mathbb{R})$ acts on $\mathbb{P}\left(\mathbb{C}^{n}\right)$ via complex conjugation. Similarly, let $\mathbb{H}=\mathbb{C} \oplus \mathbb{C}$ j denote the quaternions, and let $\mathbb{P}\left(\mathbb{H}^{n}\right)$ be the projective space of complex 1-dimensional subspaces of $\mathbb{H}^{n}$. We give $\mathbb{P}\left(\mathbb{H}^{n}\right)$ the $\mathbb{Z} / 2$-action induced by multiplication by $\jmath$ on the left of $\mathbb{H}^{n}$. As a space, $\mathbb{P}\left(\mathbb{H}^{n}\right)$ is homeomorphic to $\mathbb{P}\left(\mathbb{C}^{2 n}\right)$; however the $\mathbb{Z} / 2$-actions on $\mathbb{P}\left(\mathbb{C}^{2 n}\right)$ and $\mathbb{P}\left(\mathbb{H}^{n}\right)$ are quite distinct. In fact, these spaces are the complex-valued points of the two inequivalent Brauer-Severi schemes of rank $2 n-1$ over $\mathbb{R}$, under the action of the Galois group. The aforementioned classifying spaces $(\mathbb{Z} \times B U)_{\mathbb{R}}$ and $(\mathbb{Z} \times B U)_{\mathbb{H}}$ are then constructed using the usual equivariant stabilization of the Grassmannians of complex linear subspaces of $\mathbb{P}\left(\mathbb{C}^{n}\right)$ and $\mathbb{P}\left(\mathbb{H}^{n}\right)$, respectively.

In order to develop a theory of characteristic classes for $K R^{*}$ and $K H^{*}$, one needs to introduce the appropriate equivariant cohomology theories. In Kah87 B. Kahn defined characteristic classes for Real bundles, taking values in GaloisGrothendieck cohomology with coefficients in the $\mathbb{Z} / 2$-modules $\mathbb{Z}(n)$. In the case of quaternionic bundles, Dupont posed in Dup99 the question of which equivariant cohomology theory would be the natural target of characteristic classes, but the question was left unanswered.

In this paper we provide an answer to this question by constructing a cohomology theory $\mathfrak{Z}_{\mathbb{H}}^{*}$ in which the characteristic classes for quaternionic bundles take their values. Furthermore, we extend these characteristic classes to a natural transformation of $R O(\mathbb{Z} / 2)$-graded cohomology theories $K H^{*} \rightarrow \mathfrak{Z}_{\mathbb{H}}^{*}$. A crucial aspect of our construction is the fact that the two distinct Brauer-Severi varieties $\mathbb{P}\left(\mathbb{C}^{n}\right)$ and $\mathbb{P}\left(\mathbb{H}^{n}\right)$, used in the construction of the classifying spaces for $K R^{*}$ and $K H^{*}$, are also used to construct classifying spaces for the corresponding cohomology theories. Under this approach, the classifying maps for the characteristic classes have a similar description in both cases.

In order to place our constructions under the proper perspective, let us describe how the characteristic classes for Real bundles were extended to $K R^{*}$ in [LLFM98b] and [dS03a]. The main constructions go back to [LM91], [BLLF93] and [LLFM96].

Let $Z^{q}\left(\mathbb{P}\left(\mathbb{C}^{n}\right)\right)$ denote the group of algebraic cycles of codimension $q$ in $\mathbb{P}\left(\mathbb{C}^{n}\right)$. This is an abelian topological group on which $\operatorname{Gal}(\mathbb{C} / \mathbb{R})$ acts via topological automorphisms. See [LLFM98a] for details and additional references. The equivariant homotopy type of $z^{q}\left(\mathbb{P}\left(\mathbb{C}^{n}\right)\right)$ was determined in dS03a, and it turns out to be a product of classifying spaces for equivariant coohomology with coefficients in the constant Mackey functor $\underline{\mathbb{Z}}$. More precisely, one has a canonical equivariant homotopy equivalence

$$
Z^{q}\left(\mathbb{P}\left(\mathbb{C}^{n}\right)\right) \cong \mathbb{Z} \times K(\mathbb{Z}(1), 2) \times K(\mathbb{Z}(2), 4) \times \cdots \times K(\mathbb{Z}(q), 2 q) .
$$

The associated equivariant cohomology theory is $R O(\mathbb{Z} / 2)$-graded (bigraded, in this case) and the resulting invariants arise naturally in $\mathbb{Z} / 2$-homotopy theory. The 
functor represented by $K(\mathbb{Z}(q), 2 q)$ is denoted $H^{2 q, q}(-; \underline{\mathbb{Z}})$. See Section 1.3 for notation.

The situation in $K R$-theory follows a standard, albeit non-trivial, pattern. A canonical stabilization $\lim _{\longrightarrow} G r^{q}\left(\mathbb{C}^{n}\right)$ in the $\mathbb{Z} / 2$-homotopy category, of Grassmannians as $\operatorname{Gal}(\mathbb{C} / \mathbb{R})$-spaces, produces a classifying space $B U_{\mathbb{R}}$ for $K R$-theory. It is easy to see that $H^{*, *}\left(B U_{\mathbb{R}} ; \underline{\mathbb{Z}}\right)$ is a polynomial ring over $H^{*, *}(p t ; \underline{\mathbb{Z}})$ on certain characteristic classes, the equivariant Chern classes for $K R$-bundles. Furthermore, the inclusion of $G r^{q}\left(\mathbb{C}^{n}\right) \hookrightarrow Z^{q}\left(\mathbb{P}\left(\mathbb{C}^{n}\right)\right)$ stabilizes to give a map of equivariant infinite loop spaces

$$
\mathbf{c}_{\mathbb{R}}: B U_{\mathbb{R}} \rightarrow Z \cong \prod_{p} K(\mathbb{Z}(p), 2 p),
$$

which classifies the total Chern class.

In this paper we provide the quaternionic counterpart of the constructions in $K R^{*}$-theory described above. This turns out to be a more subtle issue, and our answer was inspired by the following observation, made in Ati66] and Dup69. If $X$ is a Real space, then one has an isomorphism:

$$
K R(X \times \mathbb{P}(\mathbb{H})) \cong K R(X) \oplus K H(X) .
$$

The first task is to determine the $\mathbb{Z} / 2$-homotopy type of $Z^{q}\left(\mathbb{P}\left(\mathbb{H}^{n}\right)\right)$, under the $\mathbb{Z} / 2$-action induced by $\jmath$, as explained above. This is the action induced by the $\operatorname{Gal}(\mathbb{C} / \mathbb{R})$ action on the complex points $\mathbb{P}\left(\mathbb{H}^{n}\right)$ of the Brauer-Severi variety of rank $n-1$ over $\mathbb{R}$. This problem was first considered in [LLFM98b], where it was proved that quaternionic suspension, $\mathbb{Z}_{\mathbb{H}}: \mathcal{Z}^{q}\left(\mathbb{P}\left(\mathbb{H}^{n}\right)\right) \rightarrow \mathcal{Z}^{q}\left(\mathbb{P}\left(\mathbb{H}^{n+1}\right)\right)$, is a $\mathbb{Z} / 2$-homotopy equivalence. This reduced the problem to computing the $\mathbb{Z} / 2$-homotopy type of cycle spaces of dimensions 0 and 1 . Their homotopy type is quite distinct, and this somehow reflects the sharp difference between quaternionic bundles of even and odd complex rank. Later, in [LLFM98c the homotopy type of the space of cycles $Z^{q}\left(\mathbb{P}\left(\mathbb{H}^{n}\right)\right)^{\mathbb{Z} / 2}$ was computed using suspension to a Real bundle other than $\mathcal{O}(1)$ (which corresponds to complex suspension).

Based on the techniques of [LLFM98c], and on the $\mathbb{Z} / 2$-equivariant perspective of dS03a, we first establish the following splitting result.

Theorem 3.4, For $k<n$ there are canonical equivariant homotopy equivalences:

$$
z^{2 k-1}\left(\mathbb{P}\left(\mathbb{H}^{n}\right)\right) \cong \prod_{j=1}^{k} F\left(\mathbb{P}(\mathbb{H})_{+}, K(\mathbb{Z}(2 j-1), 4 j-2)\right)
$$

and

$$
Z^{2 k}\left(\mathbb{P}\left(\mathbb{H}^{n}\right)\right) \cong \prod_{j=0}^{k} F\left(\mathbb{P}(\mathbb{H})_{+}, K(\mathbb{Z}(2 j), 4 j)\right)
$$

where $F(-,-)$ denotes based maps.

Note that the spaces $F\left(\mathbb{P}(\mathbb{H})_{+}, K(\mathbb{Z}(q), 2 q)\right)$ are classifying spaces for the cohomology functors $H^{2 q, q}(-\times \mathbb{P}(\mathbb{H}) ; \underline{\mathbb{Z}})$. This result completely determines the $\mathbb{Z} / 2$ homotopy type of $Z^{m}\left(\mathbb{P}\left(\mathbb{H}^{n}\right)\right)$.

Applying a suitable stabilization procedure to the spaces $Z^{q}\left(\mathbb{P}\left(\mathbb{H}^{n}\right)\right)$ we obtain a Real space $\boldsymbol{Z}_{\mathbb{H}}$. The connected components of this space are products of classifying spaces for the functors $H^{2 *, *}(-\times \mathbb{P}(\mathbb{H}) ; \underline{\mathbb{Z}})$, according to the splitting of Theorem 3.4 
Theorem 3.7. The space $\mathcal{Z}_{\mathbb{H}}$ is written as a disjoint union of connected spaces

$$
z_{\mathbb{H}}=\coprod_{j=-\infty}^{\infty} z_{\mathbb{H}}^{j}
$$

where the equivariant homotopy type of $\mathcal{Z}_{\mathbb{H}}^{j}$ is totally determined by

$$
z_{\mathbb{H}}^{j} \cong \begin{cases}\left(\prod_{k=1}^{\infty} F\left(\mathbb{P}(\mathbb{H})_{+}, K(\mathbb{Z}(2 k-1), 4 k-2)\right)\right)_{1} & \text { if } j \text { is odd }, \\ \prod_{k=1}^{\infty} F\left(\mathbb{P}(\mathbb{H})_{+}, K(\mathbb{Z}(2 k), 4 k)\right) & \text { if } j \text { is even } .\end{cases}
$$

Using standard results in equivariant homotopy theory (cf. CW91]), we prove that the complex join pairing on algebraic cycles induces an equivariant infinite loop space structure on $\boldsymbol{Z}_{\mathbb{H}}$. We denote by $\mathfrak{Z}_{\mathbb{H}}^{*}$ the resulting equivariant cohomology theory. Even at the zero-th level, $\mathfrak{Z}_{\mathbb{H}}^{0}(X)$ has a non-trivial structure, coming from the $H$-space structure on $Z_{\mathbb{H}} ;$ cf. Proposition 5.7 .

A similar stabilization procedure is then applied to the Grassmannians $G^{q}\left(\mathbb{H}^{n}\right)$ and the result is an equivariant infinite loop space $(\mathbb{Z} \times B U)_{\mathbb{H}}=\coprod_{j \in \mathbb{Z}} B U_{\mathbb{H}}^{j}$ which classifies $K H^{*}$, as described above. The inclusion $\mathrm{Gr}^{q}\left(\mathbb{H}^{n}\right) \subset Z^{q}\left(\mathbb{P}\left(\mathbb{H}^{n}\right)\right)$ induces a total Chern class map $c^{\mathbb{H}}:(\mathbb{Z} \times B U)_{\mathbb{H}} \rightarrow \mathcal{Z}_{\mathbb{H}}$, which turns out to be a map of equivariant infinite loop spaces; cf. (48).

In order to understand this Chern class map, we compute the equivariant cohomology group $\mathfrak{Z}_{\mathbb{H}}^{0}\left((\mathbb{Z} \times B U)_{\mathbb{H}}\right)$ by introducing suitable classes $\mathbf{c}_{k}^{\mathbb{H}} \in$ $H^{2 k, k}\left(\mathrm{BU}_{\mathbb{H}}^{2 n} \times \mathbb{P}(\mathbb{H}) ; \underline{\mathbb{Z}}\right)$ and proving the following.

Theorem 5.3. Let $\mathbf{c}_{k}^{\mathbb{H}}$ be the classes defined in (5.1). Then we have a ring isomorphism

$$
H^{*, *}\left(B U_{\mathbb{H}}^{e v} \times \mathbb{P}(\mathbb{H}) ; \underline{\mathbb{Z}}\right) \cong H^{*, *}(\mathbb{P}(\mathbb{H}) ; \underline{\mathbb{Z}})\left[\mathbf{c}_{1}^{\mathbb{H}}, \mathbf{c}_{2}^{\mathbb{H}}, \ldots, \mathbf{c}_{k}^{\mathbb{H}}, \ldots\right]
$$

The cohomology ring $H^{*, *}(\mathbb{P}(\mathbb{H}) ; \underline{\mathbb{Z}})$ is computed in Section 1.7 .

The total Chern class map $c_{\mathbb{H}}: \mathrm{BU}_{\mathbb{H}}=\coprod_{j \in \mathbb{Z}} B U_{\mathbb{H}}^{j} \rightarrow z_{\mathbb{H}}=\bigsqcup_{j \in \mathbb{Z}} z_{\mathbb{H}}^{j}$ sends the component $B U_{\mathbb{H}}^{j}$ to the component $z_{\mathbb{H}}^{j}$. Its equivariant homotopy type is determined by the following result.

Theorem 5.6. The equivariant cohomology classes determined by total quaternionic Chern class map $c^{\mathbb{H I}}$ and the splitting (40) of Theorem 3.7 are given by

$$
\begin{aligned}
\mathbf{1}+\mathbf{c}_{2}^{\mathbb{H}}+\mathbf{c}_{4}^{\mathbb{H}}+\cdots+\mathbf{c}_{2 n}^{\mathbb{H}}+\cdots & \text { on } B U_{\mathbb{H}}^{e v}, \\
\mathbf{c}_{1}^{\mathbb{H}}+\mathbf{c}_{3}^{\mathbb{H}}+\cdots+\mathbf{c}_{2 n+1}^{\mathbb{H}}+\cdots & \text { on } B U_{\mathbb{H}}^{\text {odd }} .
\end{aligned}
$$

This paper is organized as follows: in Section 1 we introduce the necessary background from $\mathbb{Z} / 2$-homotopy theory needed to state our results. In Section 2 we establish a canonical splitting for the space of zero cycles on $\mathbb{P}\left(\mathbb{H}^{n}\right)$. In Section 3 we compute the $\mathbb{Z} / 2$-homotopy type of $Z^{q}\left(\mathbb{P}\left(\mathbb{H}^{n}\right)\right)$ and define the infinite loop space of stabilized cycles $\boldsymbol{z}_{\mathbb{H}}$. In Section 4 we apply the same stabilization procedure to the Grassmannians $G^{q}\left(\mathbb{H}^{n}\right)$, obtaining an equivariant infinite loop space $(\mathbb{Z} \times B U)_{\mathbb{H}}$. We show that $(\mathbb{Z} \times B U)_{\mathbb{H}}$ classifies Dupont's quaternionic $K$ theory. Section $[5$ is dedicated to computations involving the characteristic classes for quaternionic bundles defined in Section 4 a projective bundle formula is proved and the characteristic classes for the universal quaternionic bundle over $(\mathbb{Z} \times B U)_{\mathbb{H}}$ are computed. 


\section{Preliminary Results from equivariant homotopy theory}

In this section we review definitions and results from equivariant homotopy theory. Throughout this section $G$ is an arbitrary finite group, and later on we specialize to the case $G=\mathbb{Z} / 2$.

Definition 1.1. If $V$ is a representation of $G, S^{V}$ denotes the one point compactification of $V$ and, for a based $G$-space $X, \Omega^{V} X$ denotes the space of based maps $F\left(S^{V}, X\right)$ equipped with its standard $G$-space structure. The set of equivariant based homotopy classes $\left[S^{V}, X\right]_{G}$ is denoted by $\pi_{V}(X)$. Given a $G$-space $X$, we denote by $X_{+}$the pointed $G$-space $X \cup\{+\}$, where + is a point fixed by $G$. A based $G$-space $X$ is an equivariant infinite loop space if for each orthogonal $G$-module $V$ one can find $G$-spaces $X_{V}$ along with equivariant homeomorphisms $X \cong \Omega^{V} X_{V}$ satisfying natural coherence conditions. We refer the reader to May96 for details.

1.1. Coefficient systems and Mackey functors. Let $G \mathcal{F}$ be the category of finite $G$-sets and $G$-maps. The coefficients for ordinary equivariant (co)homology are (contravariant) covariant functors from $G \mathcal{F}$ to the category $\mathcal{A} b$ of abelian groups which send disjoint unions to direct sums.

Given a contravariant coefficient system $M$ there are Bredon cohomology groups $H^{*}(-; M)$ with coefficients in $M$. They satisfy $G$-homotopy invariance and the suspension axiom, and they are classical cohomology theories in the sense that they satisfy the dimension axiom $H^{0}(p t ; M)=M$ and $H^{n}(p t ; M)=0$, for $n>0$.

There are certain coefficient systems - called Mackey functors - for which Bredon cohomology can be extented to an $R O(G)$-graded theory. A Mackey functor $M$ is a pair $\left(M_{*}, M^{*}\right)$ of functors $M_{*}: G \mathcal{F} \rightarrow \mathcal{A} b$ and $M^{*}: G \mathcal{F}^{o p} \rightarrow \mathcal{A} b$ with the same value on objects and which transform each pull-back diagram

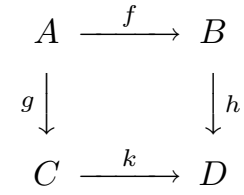

in $G \mathcal{F}$ into a commutative diagram in $\mathcal{A} b$

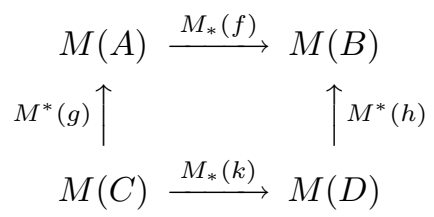

Example 1.2. In this paper we are interested in the case where $M=\underline{\mathbb{Z}}$ is the Mackey functor constant at $\mathbb{Z}$. This Mackey functor is uniquely determined by the following conditions (cf. May86, Prop. 9.10]):

(i) $\underline{\mathbb{Z}}(G / H)=\mathbb{Z}$, for $H \leq G$;

(ii) if $K \leq H$, the value of the contravariant functor $\underline{\mathbb{Z}}^{*}$ on the projection $\rho$ : $G / K \rightarrow G / H$ is the identity.

The $R O(G)$-graded cohomology groups with coefficients in a Mackey functor $M$ are denoted $H^{*}(-; M)$ and the corresponding reduced cohomology groups are denoted $\widetilde{H}^{*}(-; M)$. For each real orthogonal representation $V$ there is a classifying space $K(M, V)$ such that, for any $G$-space $X$,

$$
H^{V}(X ; M) \cong\left[X_{+}, K(M, V)\right]_{G} .
$$


The spaces $K(M, V)$ come with $G$-homotopy equivalences

$$
K(M, V) \cong \Omega^{W} K(M, V+W)
$$

which fit together to form the equivariant Eilenberg-Mac Lane spectrum $H M$. This implies that $H^{*}(-; M)$ satisfies the suspension axiom in the direction of any representation:

$$
\widetilde{H}^{V+W}\left(S^{V} \wedge X ; M\right) \cong \widetilde{H}^{W}(X ; M) .
$$

1.2. Dold-Thom theorem. Our interest in the Mackey functor $\underline{\mathbb{Z}}$ lies in the fact that just as the space of zero cycles (of degree zero) on the sphere $S^{n}$ is a model for the non-equivariant Eilenberg-MacLane space $K(\mathbb{Z}, n)$, zero cycles on a representation sphere $S^{V}$ provide a model for $K(\underline{\mathbb{Z}}, V)$. This is a consequence of the following equivariant version of the classical Dold-Thom theorem.

Notation 1.3. Let $X$ be a $G$-space. The topological group of zero cycles on $X$, denoted by $z_{0}(X)$, comes with an augmentation deg $: z_{0}(X) \rightarrow \mathbb{Z}$ sending an element $\sum_{i} n_{i} x_{i} \in z_{0}(X)$ to $\sum_{i} n_{i} \in \mathbb{Z}$. Denote by $z_{0}(X)_{\mathrm{o}}$ the kernel of this augmentation.

Theorem 1.4 (dS03b). Let $G$ be a finite group, let $X$ be a based $G$ - $C W$-complex and let $V$ be a finite dimensional $G$-representation. Then there is a natural equivalence

$$
\pi_{V} A G(X) \cong \widetilde{H}_{V}^{G}(X ; \underline{\mathbb{Z}}) .
$$

In particular, $A G\left(S^{V}\right)$ is a $K(\underline{\mathbb{Z}}, V)$ space.

Remark 1.5. We would like to thank the referee for having pointed out that long ago G. Segal, in an unpublished work, proved a version of the equivariant Dold-Thom theorem.

1.3. Motivic notation. From now on we restrict ourselves to the case of $G=\mathbb{Z} / 2$. We will use motivic notation for $\mathbb{Z} / 2$-equivariant cohomology, for it is compatible with the invariants used in algebraic geometry.

Notation 1.6. Let $\mathbf{s}$ be the one dimensional real sign representation of $\mathbb{Z} / 2$ and let $\mathbf{1}$ stand for the one dimensional trivial representation. Then $R O(\mathbb{Z} / 2)=\mathbb{Z} \cdot \mathbf{1}+\mathbb{Z} \cdot \mathbf{s}$. With $p \geq q$, we write

(1) $\mathbb{R}^{p, q}$ for $(p-q) \cdot \mathbf{1} \oplus q \cdot \mathbf{s}$;

(2) $S^{p, q}$ for $\mathbb{R}^{p, q} \cup\{\infty\}$;

(3) $H^{p, q}(-; \underline{\mathbb{Z}})$ for $H^{\mathbb{R}^{p, q}}(-; \underline{\mathbb{Z}})$;

(4) $K(\mathbb{Z}(q), p)$ for $K\left(\underline{\mathbb{Z}}, \mathbb{R}^{p, q}\right)$.

1.4. Vector bundles over Real spaces. Given a Real space $X$, one can use its equivariant structure to study two classes of complex bundles over $X$, namely the Real and quaternionic bundles. This study yields two distinct, albeit related, equivariant theories: the Real K-theory studied by Atiyah in [Ati66] and the (equivariant) quaternionic $K$-theory studied by Dupont in Dup69.

Definition 1.7. Let $(X, \sigma)$ be a Real space and $p: E \rightarrow X$ be a complex vector bundle. Let $\tau: E \rightarrow E$ be a continuous map covering $\sigma$ and such that for any $x \in X$ the resulting map $\tau: E_{x} \rightarrow E_{\sigma x}$ is anti-linear.

(a) If $\tau^{2}=$ id then $(E, \tau)$ is a Real bundle;

(b) If $\tau^{2}=-$ id then $(E, \tau)$ is a quaternionic bundle. 
The dimension of a Real or quaternionic bundle is defined as its complex dimension, and morphisms between such bundles are bundle morphisms that commute with the structure maps $\tau$.

The isomorphism classes of Real bundles form a monoid under Whitney sum, whose Grothendieck group is called the Real $K$-theory $K R^{0}(X)$ of $X$. Similarly, the Grothendieck group of isomorphism classes of quaternionic bundles gives the quaternionic $K$-theory groups $K H^{0}(X)$ of $X$.

It turns out that $K R(-)$ yields a $\mathbb{Z} / 2$-equivariant cohomology theory represented by a $\mathbb{Z} / 2$-spectrum whose 0 -th space is $\mathbb{Z} \times B U$ with its natural $\mathbb{Z} / 2$-action induced by complex conjugation. If one denotes the resulting Real space by $(\mathbb{Z} \times B U)_{\mathbb{R}}$, then

$$
K R(X) \cong\left[X_{+},(\mathbb{Z} \times B U)_{\mathbb{R}}\right]_{\mathbb{Z} / 2},
$$

for any compact Real space $X$. One can define Chern classes for Real bundles with values in $H^{*, *}(-; \underline{\mathbb{Z}})$ as usual, by pulling back certain classes from $H^{*, *}\left(B U_{\mathbb{R}} ; \underline{\mathbb{Z}}\right)$.

Theorem 1.8. There exist unique classes $\mathbf{c}_{n}^{\mathbb{R}} \in H^{2 n, n}\left(B U_{\mathbb{R}} ; \underline{Z}\right)$ whose image under the forgetful map to singular cohomology is the n-th Chern class $\mathbf{c}_{n} \in H^{2 n}\left(B U_{\mathbb{R}} ; \mathbb{Z}\right)$. Furthermore, we have the following ring isomorphism:

$$
H^{*, *}\left(B U_{\mathbb{R}} ; \underline{\mathbb{Z}}\right) \cong R\left[\mathbf{c}_{1}^{\mathbb{R}}, \ldots, \mathbf{c}_{n}^{\mathbb{R}}, \ldots\right],
$$

where $R$ is the cohomology ring of a point, $H^{*, *}(p t ; \underline{\mathbb{Z}})$.

Proof. This follows from the fact that $B U_{\mathbb{R}}$ has an equivariant cell decomposition given by the Schubert cells.

Definition 1.9. The $n$-th equivariant Chern class of a virtual Real bundle $\xi$ over $X$, classified by $f: X \rightarrow B U_{\mathbb{R}}$, is the cohomology $\operatorname{class} c_{n}^{\mathbb{R}}(\xi):=f^{*}\left(\mathbf{c}_{n}^{\mathbb{R}}\right) \in H^{2 n, n}(X ; \underline{\mathbb{Z}})$. The equivariant Chern classes satisfy Whitney sum and projective bundle formulas.

In Sections 4 and 5 we develop corresponding results for quaternionic $K$-theory.

1.5. Thom isomorphism for Real bundles. Just as in non-equivariant homotopy theory, the existence of a Thom isomorphism is directly related to the existence of orientations in the cohomology theory under study. We will see that Real bundles are $\mathrm{H} \underline{Z}$-orientable and hence there is a corresponding Thom isomorphism for Real bundles.

Definition 1.10 (May96|). Let $G$ be a finite group. Let $\xi \stackrel{p}{\rightarrow} X$ be an $n$-plane $G$-bundle over a $G$-space $X$. An $\mathrm{H} \underline{\mathbb{Z}}$-orientation of $\xi$ is an element $\mu$ of $\mathrm{H}_{\mathbb{Z}^{\alpha}}(\mathrm{T}(X))$ for some $\alpha$ of virtual dimension $n$, such that, for each inclusion $i: G / H \rightarrow X$, the restriction $i^{*} \mu$ to $\mathrm{T}\left(i^{*} \xi\right)$ is a generator of the free $\mathrm{H}_{\underline{\mathbb{Z}}}^{*}\left(S^{0}\right)$-module $\mathrm{H} \underline{\mathbb{Z}}_{H}^{*}\left(\mathrm{~T}\left(i^{*} \xi\right)\right)$.

Proposition 1.11. Let $X$ be a Real space and let $\xi \stackrel{p}{\rightarrow} X$ be a Real bundle. Then $\xi$ is $\mathrm{H} \underline{\mathbb{Z}}$-orientable.

Proof. It suffices to consider the case where $\xi$ is a Real line bundle. In this case we observe that $\mathrm{T}(\xi)=\mathbb{P}(\xi \oplus \mathbb{C}) / \mathbb{P}(\xi)$ and set $\mu=c_{1}(\lambda)$ where $\lambda \rightarrow \mathbb{P}(\xi \oplus \mathbb{C})$ is the tautological line bundle and $c_{1}$ denotes the Real first Chern class. Since $\left.\lambda\right|_{\mathbb{P}(\xi)}$ is trivial, $\mu$ descends to a class in the cohomology of $\mathrm{T}(\xi)$, which is also denoted by $\mu$. Consider an equivariant map $i:(\mathbb{Z} / 2) / H \rightarrow X$. There are two possible cases. 
(i) $H=\mathbb{Z} / 2$ : In this case $\mathrm{T}\left(i^{*} \xi\right)=\mathbb{P}\left(\mathbb{C}^{2}\right)$ with the $\mathbb{Z} / 2$-action given by complex conjugation. Then $\left.c_{1}(\lambda)\right|_{\mathbb{P}\left(\mathbb{C}^{2}\right)}$ is the first Chern class of the tautological bundle over $\mathbb{P}\left(\mathbb{C}^{2}\right)$, which is a generator for $H^{* * *}\left(\mathbb{P}\left(\mathbb{C}^{2}\right), \underline{\mathbb{Z}}\right)$ over $\mathrm{H} \underline{\mathbb{Z}}^{*}\left(S^{0}\right)=$ $H^{*, *}(p t, \underline{\mathbb{Z}})$.

(ii) $H=\{0\}$. In this case $\mathrm{T}(\xi)=\mathbb{Z} / 2_{+} \wedge S^{2,0}$. We have $H^{*, *}\left(\mathbb{Z} / 2_{+} \wedge S^{2,0}, \underline{\mathbb{Z}}\right) \cong$ $H^{*}\left(S^{2} ; \mathbb{Z}\right)$ and $K R\left(\mathbb{Z} / 2_{+} \wedge S^{2,0}\right) \cong K\left(S^{2}\right)$. It is easy to see that, under these isomorphisms, $c_{1}(\lambda)$ is the usual Chern class and hence it generates $H^{*, *}\left(\mathbb{Z} / 2_{+} \wedge S^{2,0}, \underline{\mathbb{Z}}\right)$.

Definition 1.12. Given a vector bundle $\xi \rightarrow X$ over $X$, the projection $p: \mathbb{P}(\xi \oplus 1)$ $\rightarrow X$ along with the quotient map $q: \mathbb{P}(\xi \oplus 1) \rightarrow \mathrm{T}(\xi)=\mathbb{P}(\xi \oplus 1) / \mathbb{P}(\xi)$ induces a map $\Delta: \mathrm{T}(\xi) \rightarrow \mathrm{T}(\xi) \wedge X_{+}$called the Thom diagonal of $\xi$.

Proposition 1.13 (Thom isomorphism for Real bundles). Let $\xi \stackrel{p}{\rightarrow} X$ be a Real $n$-bundle over a Real space $X$ and let $\mu \in H^{2 n, n}(\mathrm{~T}(\xi), \underline{\mathbb{Z}})$ be an orientation for $\xi$. Then

$$
\cup \mu: H^{p, q}\left(X_{+}, \underline{\mathbb{Z}}\right) \longrightarrow \widetilde{H}^{2 n+p, n+q}(\mathrm{~T}(\xi), \underline{\mathbb{Z}})
$$

is an isomorphism for all $p, q$. Furthermore, there is an equivariant homotopy equivalence

$$
\phi_{\mu}: Z_{0}(\mathrm{~T}(\xi))_{\mathrm{o}} \stackrel{\cong}{\longrightarrow} z_{0}\left(X_{+} \wedge S^{2 n, n}\right)_{\mathrm{o}}
$$

which induces the Thom isomorphism in homology

$$
\widetilde{H}_{p, q}(\mathrm{~T}(\xi), \underline{\mathbb{Z}}) \cong H_{p-2 n, q-n}(X, \underline{\mathbb{Z}}) .
$$

Proof. The existence of the Thom isomorphism is an immediate consequence of Proposition 1.11 see May96]. We proceed to construct an explicit map $\phi_{\mu}$ at the classifying space level which induces this isomorphism. Let $f_{\mu}: \mathrm{T}(\xi) \rightarrow Z_{0}\left(S^{2 n, n}\right)_{\mathrm{o}}$ be a (based) classifying map for the orientation class $\mu$, hence $f_{\mu}(\infty)=0$. Consider the composition $\mathrm{T}(\xi) \stackrel{\Delta}{\longrightarrow} \mathrm{T}(\xi) \wedge X_{+} \stackrel{f_{\mu} \wedge i d}{\longrightarrow} \mathcal{Z}_{0}\left(S^{2 n, n}\right)_{\mathrm{o}} \wedge X_{+} \rightarrow \mathcal{Z}_{0}\left(S^{2 n, n} \wedge X_{+}\right)_{\mathrm{o}}$, where $\Delta$ is the Thom diagonal, and the last map comes from the structure of functor with smash products (FSP) for $z_{0}(-)$. This composition induces a function $\phi_{\mu}: z_{0}(\mathrm{~T}(\xi))_{\mathrm{o}} \rightarrow z_{0}\left(S^{2 n, n} \wedge X_{+}\right)_{\mathrm{o}}$.

We claim that $\phi_{\mu}$ induces the Thom isomorphism in homology. Indeed, since $z_{0}\left(S^{p, q}\right)_{\mathrm{o}}$ is a $K(\mathbb{Z}(q), p)$-space, the assignment $(p, q) \mapsto z_{0}\left(S^{p, q}\right)_{\mathrm{o}}$ gives a model for $\mathrm{H} \underline{\mathbb{Z}}$. Moreover $\wedge$ induces a pairing

$$
K(\mathbb{Z}(q), p) \wedge K\left(\mathbb{Z}\left(q^{\prime}\right), p^{\prime}\right) \longrightarrow K\left(\mathbb{Z}\left(q+q^{\prime}\right), p+p^{\prime}\right)
$$

which gives the usual ring spectrum structure on $\mathrm{H} \underline{\mathbb{Z}}$; cf. Dug01 and $\mathrm{dSO3b}$. It follows that the map in homology represented by $\phi_{\mu}$ is

$$
\widetilde{H}_{p, q}(\mathrm{~T}(\xi), \underline{\mathbb{Z}}) \ni a \stackrel{\phi_{\mu_{*}}}{\longrightarrow} p_{*}(\mu \cap a) \in H_{p-2 n, q-n}(X, \underline{\mathbb{Z}}),
$$

the usual definition of the Thom isomorphism. The proof that $\phi_{\mu *}$ is in fact an isomorphism goes exactly as in the non-equivariant case. Using the five lemma and the Mayer-Vietoris sequence it is possible to reduce to the case where $\xi \stackrel{p}{\rightarrow} X$ is the trivial bundle, in which case $\mathrm{T}(\xi) \cong X_{+} \wedge S^{2 n, n}$ and it is clear that $\phi_{\mu_{*}}$ is an isomorphism. 
1.6. Poincaré duality. A smooth manifold $X$ is called a Real $n$-manifold if it has the structure of a Real space $(X, \sigma)$ whose tangent bundle becomes a Real $n$-bundle over $(X, \sigma)$ under the action induced by $d \sigma$.

Proposition 1.14. Let $X$ be a connected Real manifold of dimension n. Then, for each $k \geq 0$, there is an equivariant homotopy equivalence

$$
\mathcal{P}: F\left(X_{+} ; z_{0}\left(S^{2(n+k), n+k}\right)_{\mathrm{o}}\right) \stackrel{\cong}{\longrightarrow} z_{0}\left(S^{2 k, k} \wedge X_{+}\right)_{\mathrm{o}},
$$

which upon passage to homotopy groups induces the Poincaré duality isomorphism

$$
H^{2 n-p, n-q}(X, \underline{\mathbb{Z}}) \cong H_{p, q}(X, \underline{\mathbb{Z}}) .
$$

Proof. By Proposition 1.13 the tangent bundle of $X$ has an orientation in degree $(2 n, n)$. It follows CW92 that $X$ satisfies Poincaré duality as in (8) and the duality isomorphism is given by cap product with the fundamental class $\mathbf{z} \in H_{2 n, n}(X, \underline{\mathbb{Z}})$ which corresponds to $1 \in H^{0,0}(X, \underline{\mathbb{Z}})=H^{0}\left(X_{\mathbb{Z} / 2} ; \mathbb{Z}\right)$; cf. [CW92].

We now define a homotopy equivalence at the classifying space level realizing the Poincaré duality isomorphism. Let $r=n+k$ and let $D$ denote the composition

$$
\begin{aligned}
z_{0}(X) \wedge F\left(X_{+}, z_{0}\left(S^{2 r, r}\right)_{\mathrm{o}}\right) & \stackrel{\Delta \wedge \mathrm{id}}{\longrightarrow} z_{0}(X) \wedge z_{0}(X) \wedge F\left(X_{+}, z_{0}\left(S^{2 r, r}\right)_{\mathrm{o}}\right) \\
& \stackrel{\operatorname{id} \wedge \epsilon}{\longrightarrow} z_{0}(X) \wedge z_{0}\left(S^{2 r, r}\right)_{\mathrm{o}} \rightarrow z_{0}\left(S^{2 r, r} \wedge X_{+}\right)_{\mathrm{o}},
\end{aligned}
$$

where $\Delta$ is the diagonal map, $\varepsilon$ is the group homomorphism induced by the evaluation map $X \wedge F\left(X_{+}, z_{0}\left(S^{2 r, r}\right)_{\mathrm{o}}\right) \rightarrow z_{0}\left(S^{2 r, r}\right)_{\mathrm{o}}$, and the last arrow comes from the structure of functor with smash products (FSP) for $Z_{0}(-)$. Composing $D$ with a classifying map $S^{2 r, r} \rightarrow Z_{0}(X)$ for the fundamental class $\mathbf{z}$ we obtain a map $S^{2 n, n} \wedge F\left(X_{+}, z_{0}\left(S^{2 r, r}\right)_{\mathrm{o}}\right) \rightarrow z_{0}\left(S^{2 r, r} \wedge X_{+}\right)_{\mathrm{o}}$, with adjoint $F\left(X_{+}, z_{0}\left(S^{2 r, r}\right)_{\mathrm{o}}\right) \rightarrow$ $\Omega^{2 n, n} \mathcal{Z}_{0}\left(S^{2 r, r} \wedge X_{+}\right)_{\mathrm{o}}$. Composing with the natural equivalence

$$
\Omega^{2 n, n} Z_{0}\left(S^{2 r, r} \wedge X_{+}\right)_{\mathrm{o}} \cong z_{0}\left(S^{2 k, k} \wedge X_{+}\right)_{\mathrm{o}},
$$

yields a map

$$
\mathcal{P}: F\left(X_{+}, z_{0}\left(S^{2(n+k), n+k}\right)_{\mathrm{o}}\right) \rightarrow z_{0}\left(S^{2 k, k} \wedge X_{+}\right)_{\mathrm{o}}
$$

which induces the cap product with $\mathbf{z}$. Hence $\mathcal{P}$ is an equivariant homotopy equivalence.

1.7. The $R O(\mathbb{Z} / 2)$-graded cohomology of the Brauer-Severi curve $\mathbb{P}(\mathbb{H})$. We start describing a relation between $H^{*, *}(-; \underline{\mathbb{Z}})$ and Galois-Grothendieck cohomology. Let $G$ be a finite group. Recall that the Borel cohomology $\hat{H}_{G}^{*}(X ; R)$ of a $G$-space $X$ with coefficients in a ring $R$ is just the ordinary cohomology of the Borel construction $X_{h G}:=X \times_{G} E G$. The Galois-Grothendieck cohomology groups of $X$, with coefficients in a $G$-sheaf $\mathcal{F}$, is defined as $\hat{H}_{G}^{*}(X ; \mathcal{F}):=H^{*}\left(X_{h G} ; \mathcal{F} \times{ }_{G} E G\right)$, where $\mathcal{F} \times{ }_{G} E G$ is seen as a $G$-sheaf over $X_{h G}$. Given any $G$-spectrum $k_{G}$ there is also a Borel-type cohomology theory associated with $k_{G}$, defined as the cohomology represented by the $G$-spectrum $F\left(E G_{+}, k_{G}\right)$.

From now on, we specialize to the case $G=\mathbb{Z} / 2$, and $k_{G}=\mathrm{H} \underline{\mathbb{Z}}$. Consider the $\mathbb{Z} / 2$-sheaves $\mathbb{Z}(n)$, which denote the constant sheaf $\mathbb{Z}$ considered as a $\mathbb{Z} / 2$-sheaf with the $\mathbb{Z} / 2$-action of multiplication by $(-1)^{n}$. The following proposition relates GaloisGrothendieck cohomology with coefficients in the sheaves $\mathbb{Z}(n)$ to the cohomology represented by $F\left(E \mathbb{Z} / 2_{+}, \mathrm{H} \underline{Z}\right)$. 
Proposition 1.15. Let $p, q$ be non-negative integers such that $p \geq q$. There are natural isomorphisms

$$
\Psi: H^{p, q}\left(-\times E \mathbb{Z} / 2_{+} ; \underline{\mathbb{Z}}\right) \rightarrow \hat{H}_{\mathbb{Z} / 2}^{p}(-; \mathbb{Z}(q)),
$$

which assemble into a ring homomorphism

$$
\Psi: H^{*, *}\left(-\times E \mathbb{Z} / 2_{+} ; \underline{\mathbb{Z}}\right) \rightarrow \hat{H}_{\mathbb{Z} / 2}^{*}(-; \mathbb{Z}(*)) .
$$

In particular, if $X$ is a free $\mathbb{Z} / 2$-space, $H^{*, *}(X ; \underline{\mathbb{Z}})$ is periodic with period $(0,2)$, and the periodicity isomorphism is given by multiplication with a generator of $H^{0,2}(E \mathbb{Z} / 2 ; \underline{\mathbb{Z}}) \cong \mathbb{Z}$.

Proof. We use $Z_{0}\left(S^{p, q}\right)_{\mathrm{o}}$ as a model for the classifying space $K\left(\underline{\mathbb{Z}}, \mathbb{R}^{p, q}\right)$. Our first observation is that the cohomology groups $\hat{H}^{p}(X ; \mathbb{Z}(q))$ can be expressed as Bredon cohomology groups of $X \times E \mathbb{Z} / 2$. In fact, we have

$$
\hat{H}_{\mathbb{Z} / 2}^{p}(X ; \mathbb{Z}(q)) \cong H^{p}(X \times E \mathbb{Z} / 2 ; \underline{\mathbb{Z}}(q)),
$$

where the right-hand side denotes Bredon cohomology and $\underline{\mathbb{Z}}(q)$ is the coefficient system determined by the $\mathbb{Z} / 2$-module $\mathbb{Z}(q)$. Hence,

$$
\hat{H}_{\mathbb{Z} / 2}^{p}(X ; \mathbb{Z}(q)) \cong\left[X \times E \mathbb{Z} / 2_{+}, Z_{0}\left(S^{p}\right)_{\mathrm{o}} \otimes \mathbb{Z}(q)\right]_{\mathbb{Z} / 2},
$$

where $\mathcal{Z}_{0}\left(S^{p}\right)_{\mathrm{o}} \otimes \mathbb{Z}(q)$ is $\mathcal{Z}_{0}\left(S^{p}\right)_{\mathrm{o}}$ with the $\mathbb{Z} / 2$-action given by multiplication by $(-1)^{q}$.

A direct computation shows that there is a class $\alpha_{p, q}$ in $\hat{H}_{\mathbb{Z} / 2}^{p}\left(S^{p, q} ; \mathbb{Z}(q)\right)$, whose image under the forgetful map to singular cohomology is the fundamental class of $S^{p, q}$. Hence there is an equivariant map $z_{0}\left(S^{p, q}\right)_{\mathrm{o}} \times E \mathbb{Z} / 2 \rightarrow Z_{0}\left(S^{p}\right)_{\mathrm{o}} \otimes \mathbb{Z}(q)$ which is a non-equivariant homotopy equivalence. Composition with this map induces an equivariant homotopy equivalence

$$
F\left(E \mathbb{Z} / 2_{+}, Z_{0}\left(S^{p, q}\right)_{\mathrm{o}} \times E \mathbb{Z} / 2\right) \cong F\left(E \mathbb{Z} / 2_{+}, Z_{0}\left(S^{p}\right)_{\mathrm{o}} \otimes \mathbb{Z}(q)\right) .
$$

Composing with the map $E \mathbb{Z} / 2 \rightarrow *$ gives an equivariant homotopy equivalence

$$
F\left(E \mathbb{Z} / 2_{+}, Z_{0}\left(S^{p, q}\right)_{\mathrm{o}} \times E \mathbb{Z} / 2\right) \cong F\left(E \mathbb{Z} / 2_{+}, Z_{0}\left(S^{p, q}\right)\right)_{\mathrm{o}},
$$

which induces the isomorphism $\Psi$ in (9).

The pairing $\mathbb{Z}(q) \otimes \mathbb{Z}\left(q^{\prime}\right) \rightarrow \mathbb{Z}\left(q+q^{\prime}\right)$ gives

$$
\hat{H}_{\mathbb{Z} / 2}^{p}(-; \mathbb{Z}(q)) \otimes \hat{H}_{\mathbb{Z} / 2}^{p}\left(-; \mathbb{Z}\left(q^{\prime}\right)\right) \rightarrow \hat{H}_{\mathbb{Z} / 2}^{p}\left(-; \mathbb{Z}\left(q+q^{\prime}\right)\right),
$$

and this corresponds under the natural isomorphism (9) to the cup product in $H^{*, *}\left(-\times E \mathbb{Z} / 2_{+} ; \underline{Z}\right)$, since $\alpha_{p, q} \cup \alpha_{p^{\prime}, q^{\prime}}=\alpha_{p+p^{\prime}, q+q^{\prime}}$. Now, let $t$ be a generator of $H^{0,2}(E \mathbb{Z} / 2 ; \underline{\mathbb{Z}}) \cong \mathbb{Z}$ and let $X$ be a free $\mathbb{Z} / 2$-space. The $\mathbb{Z} / 2$-homotopy equivalence $X \cong X \times E \mathbb{Z} / 2$ makes the cohomology of $X$ into a module over $H^{*, *}(E \mathbb{Z} / 2 ; \underline{Z})$, and the compatibility of $\Psi$ with products shows that the following diagram commutes:

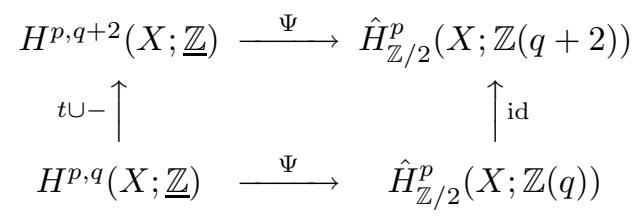

Hence $t \cup-$ is an isomorphism. 
Notation 1.16. As observed above, if $X$ is a free $\mathbb{Z} / 2$-space, there is a natural homomorphism

$$
H^{*, *}(X \times E \mathbb{Z} ; \underline{\mathbb{Z}}) \rightarrow H^{*, *}(X ; \underline{\mathbb{Z}})
$$

making $H^{*, *}(X ; \underline{\mathbb{Z}})$ into a module over $H^{*, *}(E \mathbb{Z} / 2 ; \underline{\mathbb{Z}})$. From now on we will use $t$ to denote both a generator of $H^{0,2}(E \mathbb{Z} / 2 ; \underline{\mathbb{Z}}) \cong \mathbb{Z}$ and its image under the homomorphism above. Multiplication by $t$ induces the $(0,2)$ periodicity in $H^{*, *}(X ; \underline{\mathbb{Z}})$.

Corollary 1.17. Let $X$ be a space with a free $\mathbb{Z} / 2$-action. There is an $E_{2}$ spectral sequence

$$
H^{s}\left(B \mathbb{Z} / 2 ; \mathcal{H}^{t}(X ; \mathbb{Z}(q))\right) \Longrightarrow H^{s+t, q}(X ; \underline{\mathbb{Z}}) .
$$

Proof. It is shown in Gro57] that there is an $E_{2}$ term spectral sequence

$$
H^{s}\left(B \mathbb{Z} / 2 ; \mathcal{H}^{t}(X ; \mathbb{Z}(q))\right) \Longrightarrow \hat{H}_{\mathbb{Z} / 2}^{s+t}(X ; \underline{\mathbb{Z}}(q)) .
$$

Definition 1.18. Let $\mathbb{P}\left(\mathbb{H}^{n}\right)$ denote the projective space of complex lines through the origin in $\mathbb{H}^{n}$ endowed with the analytic topology and with the involution $\sigma$ induced by left multiplication by the unit quaternion $\jmath$ on $\mathbb{H}^{n}$. This Real space coincides with the space of complex points of a real Brauer-Severi variety under the action of $\operatorname{Gal}(\mathbb{C} / \mathbb{R})$. In particular, $\mathbb{P}(\mathbb{H})$ is the space of complex points of the real Brauer-Severi curve associated with the division algebra $\mathbb{H}=\mathbb{C} \oplus \mathbb{C}$ J.

We proceed to compute the cohomology ring $H^{*, *}(\mathbb{P}(\mathbb{H}) ; \underline{\mathbb{Z}})$. For $q=0$, we have

$$
H^{*, 0}(\mathbb{P}(\mathbb{H}) ; \underline{\mathbb{Z}})=H^{*}(\mathbb{P}(\mathbb{H}) / \mathbb{Z} / 2 ; \mathbb{Z})=H^{*}\left(\mathbb{R} \mathrm{P}^{2} ; \mathbb{Z}\right) .
$$

For $q=1$, the spectral sequence (1.17) gives

$$
H^{p, 1}(\mathbb{P}(\mathbb{H}) ; \underline{\mathbb{Z}}) \cong \begin{cases}\mathbb{Z} / 2, & p=1, \\ \mathbb{Z}, & p=2, \\ 0, & \text { otherwise. }\end{cases}
$$

Moreover, $H^{1,1}(\mathbb{P}(\mathbb{H}) ; \underline{\mathbb{Z}})$ and $H^{2,0}(\mathbb{P}(\mathbb{H}) ; \underline{\mathbb{Z}})$ are generated by the image of the homomorphism

$$
H^{*, *}(E \mathbb{Z} / 2 ; \underline{\mathbb{Z}}) \rightarrow H^{*, *}(\mathbb{P}(\mathbb{H}) ; \underline{\mathbb{Z}}) .
$$

Let $\epsilon$ denote the generator of $H^{1,1}(\mathbb{P}(\mathbb{H}) ; \underline{\mathbb{Z}})$ and let $\epsilon^{\prime}$ be its image in $H^{1}(\mathbb{P}(\mathbb{H}) ; \mathbb{Z}(1))$ under the ring homomorphism $\Psi$ of (9). One can check that $\epsilon^{\prime 2} \neq 0$ hence $\epsilon^{2}$ is the generator of $H^{2,2}(\mathbb{P}(\mathbb{H}) ; \underline{\mathbb{Z}})$.

The group $H^{2,1}(\mathbb{P}(\mathbb{H}) ; \underline{\mathbb{Z}})$ is generated by the fundamental class of $\mathbb{P}(\mathbb{H})$, which we denote by $\mathbf{x}$. Using the $(0,2)$ periodicity in Proposition [1.15 one has

$$
H^{4,2}(\mathbb{P}(\mathbb{H}) ; \underline{\mathbb{Z}}) \cong H^{4}\left(\mathbb{R P}^{2} ; \mathbb{Z}\right)=0,
$$

hence $\mathbf{x}^{2}=0$. The same argument shows that $\mathbf{x} \epsilon=\mathbf{x}^{3}=0$.

Remark 1.19. The generator $\mathbf{x}$ is the first Chern class $c_{1}^{\mathbb{R}}(\mathcal{O}(2))$ of the Real bundle $\mathcal{O}(2)$ over $\mathbb{P}(\mathbb{H})$.

Putting all these facts together and using the fact that $t \cup-$ is an isomorphism, we obtain a ring isomorphism

$$
H^{*, *}(\mathbb{P}(\mathbb{H}) ; \underline{\mathbb{Z}}) \cong \mathbb{Z}\left[\epsilon, \mathbf{x}, t, t^{-1}\right] /\left(2 \epsilon, \epsilon^{3}, \mathbf{x} \epsilon, \mathbf{x}^{2}\right),
$$

where $\epsilon, t$ and $\mathbf{x}$ have degrees $(1,1),(0,2)$ and $(2,1)$, respectively. 


\section{The $\mathbb{Z} / 2$-Homotopy TYPE OF ZERO-CYCLES}

A structure on a complex vector space $V$ is a complex anti-linear map $\sigma: V \rightarrow V$ that satisfies either $\sigma^{2}=I$ or $\sigma^{2}=-I$. In the first case, $\sigma$ is called a Real structure and the pair $(V, \sigma)$ is a Real vector space; in the latter case, $\sigma$ is called a quaternionic structure and the pair $(V, \sigma)$ is a quaternionic vector space. A morphism $f:\left(V, \sigma_{V}\right) \rightarrow\left(W, \sigma_{W}\right)$ of vector spaces with structure is a complex linear map from $V$ to $W$ commuting with the respective structures.

Any Real vector space $(V, \sigma)$ of complex dimensin $n$ is isomorphic as a vector space with structure to $\left(\mathbb{C}^{n}, \sigma_{o}\right)$, where $\sigma_{o}$ denotes the usual complex conjugation on $\mathbb{C}^{n}$. Similarly, any quaternionic vector space $(V, \sigma)$ of complex dimension $2 n$ is isomorphic to $\left(\mathbb{H}^{n}, \sigma_{o}\right)$, where $\mathbb{H}=\mathbb{C} \oplus \mathbb{C}$ j denotes the algebra of quaternions and $\sigma_{o}$ is induced by multiplication by $\jmath$ on the left of $\mathbb{H}^{n}$.

Remark 2.1. It is clear that if $(V, \sigma)$ is a quaternionic vector space, then $\sigma$ naturally induces a structure $\sigma_{d}$ on the symmetric power $\operatorname{Sym}_{d}(V)$ which is Real if $d$ is even and quaternionic if $d$ is odd. In particular, one has

$$
\left(\operatorname{Sym}_{2 k}(\mathbb{H}), \sigma_{2 k}\right) \cong\left(\mathbb{C}^{2 k+1}, \sigma_{o}\right) \text { and }\left(\operatorname{Sym}_{2 k+1}(\mathbb{H}), \sigma_{2 k+1}\right) \cong\left(\mathbb{H}^{k+1}, \sigma_{o}\right)
$$

Let $\mathbb{H}^{*}$ denote the complex dual of $\mathbb{H}$. If $f: \mathbb{H}^{*} \rightarrow \mathbb{C}$ is a non-zero linear functional we denote its zero locus by $[f] \in \mathbb{P}(\mathbb{H})$. See Definition 1.18.

The $d$-fold symmetric product $\mathrm{SP}_{d}(\mathbb{P}(\mathbb{H}))$ inherits a Real space structure $\sigma$ : $\mathrm{SP}_{d}(\mathbb{P}(\mathbb{H})) \rightarrow \mathrm{SP}_{d}(\mathbb{P}(\mathbb{H}))$ induced by the involution $\sigma$ on $\mathbb{P}(\mathbb{H})$; cf. Definition 1.18 Denote the elements of $\mathrm{SP}_{d}(\mathbb{P}(\mathbb{H}))$ multiplicatively as $\left[f_{1}\right] \cdots\left[f_{d}\right]$. Then the map sending $\left[f_{1}\right] \cdots\left[f_{d}\right]$ to $\left[f_{1} \cdots f_{d}\right]$ induces an isomorphism of Real spaces between $\mathrm{SP}_{d}(\mathbb{P}(\mathbb{H}))$ and $\mathbb{P}\left(\operatorname{Sym}_{d}(\mathbb{H})\right)$.

The canonical inclusion $i_{n, q}: \mathbb{P}\left(\mathbb{H}^{n}\right) \hookrightarrow \mathbb{P}\left(\mathbb{H}^{q}\right)$, for $n<q$, given by setting the last coordinates to zero can be described up to linear isomorphism in terms of symmetric products as follows. Let $\left[f_{o}\right] \in \mathbb{P}(\mathbb{H})$ be some point and let $\left[f_{o}^{\sigma}\right]$ denote its image under the quaternionic involution $\sigma$ and define

$$
\begin{aligned}
& i_{n, q}: \mathbb{P}\left(\mathbb{H}^{n}\right)=\mathrm{SP}_{2 n-1}(\mathbb{P}(\mathbb{H})) \rightarrow \mathbb{P}\left(\mathbb{H}^{q}\right)=\mathrm{SP}_{2 q-1}(\mathbb{P}(\mathbb{H})), \\
& {\left[f_{1}\right] \cdots\left[f_{2 n-1}\right] \mapsto\left(\left[f_{o}\right]\left[f_{o}^{\sigma}\right]\right)^{q-n} \cdot\left[f_{1}\right] \cdots\left[f_{2 n-1}\right] .}
\end{aligned}
$$

This is a morphism of Real spaces.

Following [LLFM98c], given $a<b$ we define

$$
\begin{aligned}
r_{b, a}: \mathrm{SP}_{b}(\mathbb{P}(\mathbb{H})) & \rightarrow \operatorname{SP}_{\left(\begin{array}{l}
b \\
a
\end{array}\right)}\left(\operatorname{SP}_{a}(\mathbb{P}(\mathbb{H}))\right), \\
{\left[f_{1}\right] \cdots\left[f_{b}\right] } & \mapsto \sum_{|I|=a}\left[f_{i_{1}}\right] \cdots\left[f_{i_{a}}\right]
\end{aligned}
$$

where the sum runs over all multi-indices $I=\left\{1 \leq i_{1}<\cdots<i_{a} \leq b\right\}$. Notice that $r_{b, a}$ are always morphisms of Real spaces regardless of the parities of $a$ and $b$.

In order to simplify notation, write

$$
M_{n}=Z_{0}\left(\mathrm{SP}_{2 n-1}(\mathbb{P}(\mathbb{H}))\right)=Z_{0}\left(\mathbb{P}\left(\mathbb{H}^{n}\right)\right),
$$

and let

$$
\Psi_{n}: M_{n} \rightarrow Q_{n}:=M_{n} / M_{n-1}
$$

denote the quotient map. We adopt the convention that $M_{-1}=\{0\}$. 
Now, use the morphisms $r_{b, a}$ to construct maps

$$
R_{q, n}: M_{q} \rightarrow M_{n}
$$

as follows. Given $\tau \in \mathbb{P}\left(\mathbb{H}^{q}\right)$, define for $n<q$,

$$
\begin{aligned}
R_{q, n}(\tau)= & r_{2 q-1,2 n-1}(\tau) \\
& +\sum_{j=1}^{2 n-1}(-1)^{j}\left(\begin{array}{c}
q-n+j-1 \\
j
\end{array}\right)\left(\left[f_{o}\right]^{j}+\left[f_{o}^{\sigma}\right]^{j}\right) r_{2 q-1,2 n-1-j}(\tau) .
\end{aligned}
$$

Extend $R_{q, n}$ linearly to arbitrary 0 -cycles on $\mathbb{P}\left(\mathbb{H}^{q}\right)$. Finally, for $n<q$, define $q_{q, n}: M_{q} \rightarrow Q_{n}$ as the composition $q_{q, n}=\Psi_{n} \circ R_{q, n}$ and let $q_{n, n}=\Psi_{n}$.

Proposition 2.2. Let $\left\{\left(M_{n}, Q_{n}, q_{q, n}, i_{q, n}\right) \mid q_{q, n}: M_{q} \rightarrow Q_{n}, i_{n, q}: M_{n} \hookrightarrow M_{q}, 0 \leq\right.$ $n \leq q\}$ be the collection of groups and maps defined above. Then the following assertions hold:

(a) The homomorphisms $i_{n, q}$ and $q_{q, n}$ are equivariant with respect to the $\mathbb{Z} / 2$ actions on $M_{n}$ and $Q_{n}$ induced by the quaternionic structure on $\mathbb{H}^{n}$.

(b) The sequence $M_{n-1} \stackrel{i_{n-1, n}}{\longrightarrow} M_{n} \stackrel{q_{n, n}}{\longrightarrow} Q_{n}$ is an equivariant principal fibration, for all $n$.

(c) The following diagram commutes:

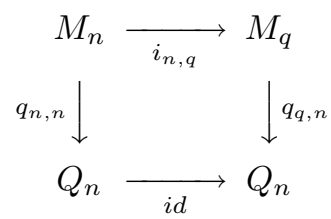

Proof. The first assertion is evident from the definitions.

Now, observe that the $\mathbb{Z} / 2$ involution on $\mathbb{P}\left(\mathbb{H}^{n}\right)$ induced by the quaternionic structure on $\mathbb{H}^{n}$ is a real analytic involution. Therefore, the pair $\left(\mathbb{P}\left(\mathbb{H}^{n}\right), \mathbb{P}\left(\mathbb{H}^{n-1}\right)\right)$ becomes a $\mathbb{Z} / 2$-simplicial pair after a suitable equivariant triangulation. Now, the second assertion follows from [LF97, Thm. 2.7]

In order to prove the last assertion, consider elements $x_{1}, \ldots, x_{2 q-1} \in \mathbb{P}(\mathbb{H})$ as free variables and let $S$ denote the polynomial ring $S:=\mathbb{Z}\left[x_{1}, \ldots, x_{2 q-1}\right]$. It is clear that $r_{2 q-1,2 n-1}\left(x_{1} \cdots x_{2 q-1}\right) \in \mathrm{SP}_{\left(\begin{array}{c}2 q-1 \\ 2 n-1\end{array}\right)}\left(\mathrm{SP}_{2 n-1}(\mathbb{P}(\mathbb{H}))\right)$ can be seen as the coefficient of $t^{2 n-1}$ in the polynomial

$$
P_{t}^{2 q-1}\left(x_{1}, \ldots, x_{2 q-1}\right):=\prod_{i=1}^{2 q-1}\left(1+x_{i} t\right) \in S[t] .
$$

In particular,

$$
r_{2 q-1,2 n-1-j} \circ i_{n, q}\left(x_{1} \cdots x_{2 n-1}\right)=r_{2 q-1,2 n-1-j}\left(x_{1} \cdots x_{2 n-1}\left(f_{o} f_{o}^{\sigma}\right)^{n-q}\right)
$$

is the coefficient of $t^{2 n-1-j}$ in the polynomial $P_{t}^{2 n-1}\left(x_{1}, \ldots, x_{2 n-1}\right)\left(1+f_{o} t\right)^{q-n}$. $\left(1+f_{o}^{\sigma} t\right)^{q-n}$. 
Using the above observations, and the definition of $R_{q, n}$, one concludes that $R_{q, n}\left(i_{n, q}\left(x_{1} \cdots x_{2 n-1}\right)\right)$ is the coefficient of $t^{2 n-1}$ in

$$
\begin{aligned}
& \left(\prod_{i=1}^{2 n-1}\left(1+x_{i} t\right)\right)\left(1+f_{o} t\right)^{q-n}\left(1+f_{o}^{\sigma} t\right)^{q-n} \\
& +\sum_{j=1}^{\infty}(-1)^{j}\left(\begin{array}{c}
q-n+j-1 \\
j
\end{array}\right)\left\{\left(f_{o}\right)^{j}+\left(f_{o}^{\sigma}\right)^{j}\right\} t^{j} \\
& \times\left(\prod_{i=1}^{2 n-1}\left(1+x_{i} t\right)\right)\left(1+f_{o} t\right)^{q-n}\left(1+f_{o}^{\sigma} t\right)^{q-n} \\
& =\left(\prod_{i=1}^{2 n-1}\left(1+x_{i} t\right)\right) \cdot\left\{\left(1+f_{o} t\right)^{q-n}\left(1+f_{o}^{\sigma} t\right)^{q-n}\right. \\
& \left.+\sum_{j=1}^{\infty}(-1)^{j}\left(\begin{array}{c}
q-n+j-1 \\
j
\end{array}\right)\left\{\left(f_{o}\right)^{j}+\left(f_{o}^{\sigma}\right)^{j}\right\} t^{j}\left(1+f_{o} t\right)^{q-n}\left(1+f_{o}^{\sigma} t\right)^{q-n}\right\}
\end{aligned}
$$

seen as an element in the ring $\mathbb{Z}\left[x_{1}, \ldots, x_{2 n-1}\right] \llbracket f_{o}, f_{o}^{\sigma}, t \rrbracket$ of formal power series in the variables $f_{o}, f_{o}^{\sigma}, t$ with coefficients in $\mathbb{Z}\left[x_{1}, \ldots, x_{2 n-1}\right]$.

We now observe that the inverse of $(1+y)^{N}$ in $\mathbb{Z} \llbracket y \rrbracket$ is given by

$$
\sum_{j=0}^{\infty}(-1)^{j}\left(\begin{array}{c}
N+j-1 \\
j
\end{array}\right) y^{j}
$$

and hence, the element

$$
\begin{aligned}
& \left(1+f_{o} t\right)^{q-n}\left(1+f_{o}^{\sigma} t\right)^{q-n} \\
& \quad+\sum_{j=1}^{\infty}(-1)^{j}\left(\begin{array}{c}
q-n+j-1 \\
j
\end{array}\right)\left\{\left(f_{o}\right)^{j}+\left(f_{o}^{\sigma}\right)^{j}\right\} t^{j}\left(1+f_{o} t\right)^{q-n}\left(1+f_{o}^{\sigma} t\right)^{q-n}
\end{aligned}
$$

in $\mathbb{Z} \llbracket f_{o}, f_{o}^{\sigma}, t \rrbracket$ is seen to be equal to

$$
\begin{aligned}
\left(1+f_{o} t\right)^{q-n}(1 & \left.+f_{o}^{\sigma} t\right)^{q-n}\left\{\left(1+f_{o} t\right)^{n-q}+\left(1+f_{o}^{\sigma} t\right)^{n-q}-1\right\} \\
& =\left(1+f_{o}^{\sigma} t\right)^{q-n}+\left(1+f_{o} t\right)^{q-n}-\left(1+f_{o} t\right)^{q-n}\left(1+f_{o}^{\sigma} t\right)^{q-n}
\end{aligned}
$$

If one writes $(1+y t)^{N}=1+y \alpha_{t}^{N}(y)$, then the right hand side of formula (21) can be written as

$$
\begin{aligned}
& \left(1+f_{o}^{\sigma} t\right)^{q-n}+\left(1+f_{o} t\right)^{q-n}-\left(1+f_{o} t\right)^{q-n}\left(1+f_{o}^{\sigma} t\right)^{q-n} \\
= & \left(1+f_{o}^{\sigma} t\right)^{q-n}+\left(1+f_{o} t\right)^{q-n}-\left(1+f_{o}^{\sigma} \alpha_{t}^{q-n}\left(f_{o}^{\sigma}\right)\right)\left(1+f_{o} \alpha_{t}^{q-n}\left(f_{o}\right)\right) \\
= & \left(1+f_{o}^{\sigma} t\right)^{q-n}+\left(1+f_{o} t\right)^{q-n} \\
& -\left\{1+f_{o}^{\sigma} \alpha_{t}^{q-n}\left(f_{o}^{\sigma}\right)+f_{o} \alpha_{t}^{q-n}\left(f_{o}\right)+f_{o}^{\sigma} f_{o} \alpha_{t}^{q-n}\left(f_{o}^{\sigma}\right) \alpha_{t}^{q-n}\left(f_{o}\right)\right\} \\
= & \left(1+f_{o}^{\sigma} t\right)^{q-n}+\left(1+f_{o} t\right)^{q-n} \\
& -\left\{\left(1+f_{o}^{\sigma} t\right)^{q-n}+\left(1+f_{o} t\right)^{q-n}-1+f_{o}^{\sigma} f_{o} \alpha_{t}^{q-n}\left(f_{o}^{\sigma}\right) \alpha_{t}^{q-n}\left(f_{o}\right)\right\} \\
=1 & -f_{o}^{\sigma} f_{o}\left\{\alpha_{t}^{q-n}\left(f_{o}^{\sigma}\right) \alpha_{t}^{q-n}\left(f_{o}\right)\right\} .
\end{aligned}
$$


We now apply the latter identity to equation (20) and obtain

$$
R_{q, n}\left(i_{n, q}\left(x_{1} \cdots x_{2 n-1}\right)\right)=x_{1} \cdots x_{2 n-1}-f_{o}^{\sigma} f_{o}(\star),
$$

for some element $\star$. Therefore,

$$
q_{q, n} \circ i_{n, q}\left(x_{1} \cdots x_{2 n-1}\right)=\Psi_{n}\left(R_{q, n}\left(i_{n, q}\left(x_{1} \cdots x_{2 n-1}\right)\right)\right)=\Psi_{n}\left(x_{1} \cdots x_{2 n-1}\right),
$$

and this shows that the diagram (18) commutes, concluding the proof.

Corollary 2.3. Given a based Real space $X$, the maps $q_{n, j}: Z_{0}\left(\mathbb{P}\left(\mathbb{H}^{n}\right)\right) \rightarrow$ $z_{0}\left(\mathbb{P}\left(\mathbb{H}^{j}\right)\right) / z_{0}\left(\mathbb{P}\left(\mathbb{H}^{j-1}\right)\right)$ induce an equivariant homotopy equivalence

$$
z_{0}\left(X \wedge \mathbb{P}\left(\mathbb{H}^{n}\right)_{+}\right)_{\mathrm{o}} \stackrel{\tilde{q}_{n, n} \times \tilde{q}_{n, n-1} \times \cdots \times \tilde{q}_{n, 1}}{\longrightarrow} \prod_{j=1}^{n} z_{0}\left(X \wedge\left\{\mathbb{P}\left(\mathbb{H}^{j}\right) / \mathbb{P}\left(\mathbb{H}^{j-1}\right)\right\}\right)_{\mathrm{o}},
$$

where we set $\mathbb{P}(\mathbb{H}) / \mathbb{P}\left(\mathbb{H}^{0}\right):=\mathbb{P}(\mathbb{H})_{+}$.

Proof. Define $M_{j}=z_{0}\left(X \wedge \mathbb{P}\left(\mathbb{H}^{j}\right)_{+}\right)_{\mathrm{o}}$ and $Q_{j}=z_{0}\left(X \wedge\left\{\mathbb{P}\left(\mathbb{H}^{j}\right) / \mathbb{P}\left(\mathbb{H}^{j-1}\right)\right\}\right)_{\mathrm{o}}$ for $j=1, \ldots, n$ and define $M_{0}=\{0\}$. Observe that one has an equivariant isomorphism $M_{j} / M_{j-1} \cong Q_{j}$ for $j=1, \ldots, n$. Let $\Psi_{j}: M_{j} \rightarrow Q_{j}$ denote the projection and let $\tilde{i}_{n, q}: M_{n} \hookrightarrow M_{q}$ be the canonical inclusion induced by the inclusion of spaces when $n<q$.

The maps $r_{2 q-1,2 n-1}$, described in (13), induce maps

$$
\tilde{r}_{2 q-1,2 n-1}: X \wedge \mathbb{P}\left(\mathbb{H}^{q}\right)_{+} \rightarrow \operatorname{SP}_{\left(\begin{array}{c}
2 q-1 \\
2 n-1
\end{array}\right)}\left(X \wedge \mathbb{P}\left(\mathbb{H}^{n}\right)_{+}\right)
$$

defined as the composition

$X \wedge \mathbb{P}\left(\mathbb{H}^{q}\right)_{+} \stackrel{i d \wedge r_{2 q-1,2 n-1}}{\longrightarrow} X \wedge\left\{\mathrm{SP}_{\left(\begin{array}{c}2 q-1 \\ 2 n-1\end{array}\right)}\left(\mathbb{P}\left(\mathbb{H}^{n}\right)\right)\right\}_{+} \stackrel{\wedge}{\longrightarrow} \mathrm{SP}_{\left(\begin{array}{c}2 q-1 \\ 2 n-1\end{array}\right)}\left(X \wedge \mathbb{P}\left(\mathbb{H}^{n}\right)_{+}\right)$,

where the latter is the natural structural map when we see $\mathrm{SP}_{*}(-)$ as a functor with smash products. Finally, define $\tilde{q}_{q, n}:=\Psi_{n} \circ \tilde{r}_{2 q-1,2 n-1}$.

It is immediate from the definitions that all the assertions in Proposition $[2.2$ hold for the new collection $\left(M_{n}, Q_{n}, \tilde{q}_{q, n}, \tilde{i}_{n, q}\right)$ above. These assertions guarantee that the spaces and maps involved, along with their restrictions to fixed point sets, satisfy the hypothesis of [FL92, Prop. 2.13]. The corollary follows.

In order to fully understand the equivariant homotopy type of $z_{0}\left(\mathbb{P}\left(\mathbb{H}^{n}\right)\right)$ we are reduced to understanding $z_{0}\left(\mathbb{P}\left(\mathbb{H}^{j}\right) / \mathbb{P}\left(\mathbb{H}^{j-1}\right)\right)_{\mathrm{o}}$. However, $\mathbb{P}\left(\mathbb{H}^{j}\right) / \mathbb{P}\left(\mathbb{H}^{j-1}\right)=$ $\mathrm{T}\left(\mathcal{O}_{\mathbb{P}(\mathbb{H})}(1) \otimes \mathbb{H}^{j-1}\right)$ is the Thom space of the Real bundle $\mathcal{O}_{\mathbb{P}(\mathbb{H})}(1) \otimes \mathbb{H}^{j-1}$. It follows from Propositions 1.13 and 1.14 that the Thom class of $\mathcal{O}_{\mathbb{P}(\mathbb{H})}(1)$, along with Poincaré duality, determines a unique equivariant homotopy equivalence

$z_{0}\left(\mathbb{P}\left(\mathbb{H}^{j}\right) / \mathbb{P}\left(\mathbb{H}^{j-1}\right)\right)_{\mathrm{o}} \cong z_{0}\left(\mathrm{~T}\left(\mathcal{O}_{\mathbb{P}(\mathbb{H})}(1) \otimes \mathbb{H}^{j-1}\right)\right)_{\mathrm{o}} \cong F\left(\mathbb{P}(\mathbb{H})_{+}, z_{0}\left(S^{4 j-2,2 j-1}\right)_{\mathrm{o}}\right)$.

This proves the following result:

Corollary 2.4. There exists a canonical equivariant homotopy equivalence

$$
\begin{aligned}
z_{0}\left(\mathbb{P}\left(\mathbb{H}^{n}\right)\right) & \cong \prod_{j=1}^{n} F\left(\mathbb{P}(\mathbb{H})_{+}, z_{0}\left(S^{4 j-1,2 j-1}\right)_{\mathrm{o}}\right) \\
& \cong \prod_{j=1}^{n} F\left(\mathbb{P}(\mathbb{H})_{+}, K(\mathbb{Z}(2 j-1), 4 j-2)\right) .
\end{aligned}
$$


The last equivalence follows from the equivariant Dold-Thom theorem proven in dS03b; cf. (1.4).

\section{Quaternionic ALgeBRAiC CYClES AND THE JOIN PAIRING}

In this section we study the equivariant topology of groups of algebraic cycles under quaternionic involution, and construct stabilizations of such objects that yield equivariant $\mathbb{Z} / 2$-spectra.

3.1. Equivariant homotopy type of algebraic cycles. Let $(V, \sigma)$ be a quaternionic vector space. An algebraic cycle of codimension $q$ on $\mathbb{P}(V)$ is a finite linear combination $\sum_{i=0}^{k} m_{i} A_{i}$, where $m_{i} \in \mathbb{Z}$ and $A_{i} \subset \mathbb{P}(V)$ is an irreducible subvariety of codimension $q$ in $\mathbb{P}(V)$. The following properties hold.

\section{Facts 3.1.}

(a) The collection of algebraic cycles of codimension $q$ in $\mathbb{P}(V)$ forms an abelian topological group $Z^{q}(\mathbb{P}(V))$ under addition of cycles; cf. [LF94].

(b) The quaternionic structure $\sigma$ on $V$ induces a continuous involution $\sigma_{*}$ : $Z^{q}(\mathbb{P}(V)) \rightarrow Z^{q}(\mathbb{P}(V))$ which is also a group homomorphism; cf. [LLFM98c. This gives an action of $\mathbb{Z} / 2$ on $Z^{q}(\mathbb{P}(V))$ via group automorphisms. We reserve the word equivariant in the present context to mean $\mathbb{Z} / 2$-equivariant under this quaternionic action.

(c) There is a continuous degree homomorphism deg: $\mathcal{Z}^{q}(\mathbb{P}(V)) \rightarrow \mathbb{Z}$ which assigns to a cycle $\sum_{i} n_{i} A_{i}$ the integer $\sum_{i} n_{i} \operatorname{deg}\left(A_{i}\right)$, where $\operatorname{deg}\left(A_{i}\right)$ is the degree of $A_{i}$ as a subvariety of $\mathbb{P}(V)$. For each $d \in \mathbb{Z}$, denote $Z^{q}(\mathbb{P}(V))_{d}:=$ $\operatorname{deg}^{-1}(d)$ the subspace of cycles of degree $d$. Each $z^{q}(\mathbb{P}(V))_{d}$ is a connected component of $Z^{q}(\mathbb{P}(V))$.

Given two quaternionic vector spaces $\left(V, \sigma_{V}\right)$ and $\left(W, \sigma_{W}\right)$, one has an equivariant external join pairing

$$
\text { \# : } Z^{q}(\mathbb{P}(V)) \times z^{q^{\prime}}(\mathbb{P}(W)) \rightarrow z^{q+q^{\prime}}(\mathbb{P}(V \oplus W))
$$

given by the ruled join of cycles. The map \# is the bilinear extension of the following operation. Given an irreducible subvariety $A \subset \mathbb{P}(V)$ of codimension $q$, and an irreducible subvariety $B \subset \mathbb{P}(W)$ of codimension $q^{\prime}$, let $A \# B$ be the irreducible subvariety of $\mathbb{P}(V \oplus W)$ obtained by taking the union of all projective lines in $\mathbb{P}(V \oplus W)$ joining points in $A$ to points in $B$, after taking the embeddings $A \subset \mathbb{P}(V)=\mathbb{P}(V \oplus 0) \subset \mathbb{P}(V \oplus W)$ and $B \subset \mathbb{P}(W)=\mathbb{P}(0 \oplus W) \subset \mathbb{P}(V \oplus W)$. We refer the reader to LLFM96] for more details.

Remark 3.2. The degree of cycles is additive with respect to addition of cycles and multiplicative with respect to the join, that is, given cycles $\sigma_{1}, \sigma_{2} \in Z^{q}(\mathbb{P}(V))$ and $\tau_{1} \in Z^{q}(\mathbb{P}(W))$, one has

$$
\operatorname{deg}\left(\sigma_{1}+\sigma_{2}\right)=\operatorname{deg} \sigma_{1}+\operatorname{deg} \sigma_{2} \quad \text { and } \quad \operatorname{deg}\left(\sigma_{1} \# \tau_{1}\right)=\operatorname{deg} \sigma_{1} \operatorname{deg} \tau_{1} .
$$

If one thinks of $\mathbb{P}(\mathbb{H})$ as an element in $Z^{0}(\mathbb{P}(\mathbb{H}))$, one can use the join to define the quaternionic suspension map:

$$
\begin{aligned}
\mathbb{Z}_{\mathbb{H}}: Z^{q}(\mathbb{P}(V)) & \rightarrow Z^{q}(\mathbb{P}(V \oplus \mathbb{H})), \\
c & \mapsto c \# \mathbb{P}(\mathbb{H}) .
\end{aligned}
$$


Remark 3.3. (1) This definition parallels the construction of the complex suspension map $\mathbb{Z}_{\mathbb{C}}: Z^{q}(\mathbb{P}(V)) \rightarrow Z^{q}(\mathbb{P}(V \oplus \mathbb{C}))$ for a Real vector space $(V, \sigma)$, whose (non-equivariant) homotopy properties were first studied in Law89]. Equivariant properties for this map, with respect to the complex conjugation involution, were studied in Lam90, LLFM98a, [LLFM98b], dS03a, Mos98.

(2) Another useful description of the suspension map is the following. Consider a surjection $f: V \rightarrow W$ of quaternionic vector spaces, and let $C \subset V$ be a (quaternionic) complement of the kernel $K:=\operatorname{ker} f$, so that $V$ is the internal direct sum $K \oplus C$ with $C \cong W$. The latter isomorphism induces a homomorphism $Z^{q}(\mathbb{P}(W)) \cong Z^{q}(\mathbb{P}(C)) \stackrel{\mathbb{\Psi}_{K}}{\longrightarrow} Z^{q}(\mathbb{P}(C \oplus K)) \cong Z^{q}(\mathbb{P}(V))$. One can easily verify that the resulting "pull-back map" $f^{*}: z^{q}(\mathbb{P}(W)) \rightarrow z^{q}(\mathbb{P}(V))$ is independent of the choice of the complement $C$.

We proceed to determine the equivariant homotopy type of spaces of algebraic cycles $\mathcal{Z}^{q}\left(\mathbb{P}\left(\mathbb{H}^{n}\right)\right)$ of arbitrary codimension $q$ on $\mathbb{P}\left(\mathbb{H}^{n}\right)$. Given a quaternionic vector space $(V, \sigma)$, the total space of the Real bundle $\pi: \mathcal{O}_{\mathbb{P}(V)}(1) \otimes \mathbb{H} \rightarrow \mathbb{P}(V)$ is $\mathbb{P}(V \oplus \mathbb{H})-\mathbb{P}(\mathbb{H})$. By the homotopy property for Real vector bundles dS03a Prop. $4.15]$ it follows that the flat pull-back of cycles

$$
\pi^{*}: z_{p}(\mathbb{P}(V)) \rightarrow z_{p+2}(\mathbb{P}(V \oplus \mathbb{H})-\mathbb{P}(\mathbb{H}))
$$

is an equivariant homotopy equivalence. Since $z_{p+2}(\mathbb{P}(\mathbb{H}))=0$ it follows that

$$
z_{p+2}(\mathbb{P}(V \oplus \mathbb{H})-\mathbb{P}(\mathbb{H})) \cong z_{p+2}(\mathbb{P}(V \oplus \mathbb{H})) .
$$

It is easy to see that, under this isomorphism, $\pi^{*}$ coincides with the suspension homomorphism (25). It follows that $\mathbb{Z}_{\mathbb{H}}$ is an equivariant homotopy equivalence.

In particular, for $k<n$, one obtains equivariant homotopy equivalences:

$$
\mathbb{Z}_{\mathbb{H}}^{n-k}: z^{2 k-1}\left(\mathbb{P}\left(\mathbb{H}^{k}\right)\right)=z_{0}\left(\mathbb{P}\left(\mathbb{H}^{k}\right)\right) \rightarrow z^{2 k-1}\left(\mathbb{P}\left(\mathbb{H}^{n}\right)\right)
$$

and

$$
\mathbb{Z}_{\mathbb{H}}^{n-k-1}: Z^{2 k}\left(\mathbb{P}\left(\mathbb{H}^{k+1}\right)\right)=z_{1}\left(\mathbb{P}\left(\mathbb{H}^{k+1}\right) \rightarrow z^{2 k}\left(\mathbb{P}\left(\mathbb{H}^{n}\right)\right) .\right.
$$

Thus, it suffices to determine the equivariant homotopy type of $z_{1}\left(\mathbb{P}\left(\mathbb{H}^{k+1}\right)\right)$.

Again, let $(V, \sigma)$ be a quaternionic vector space, and recall that $\operatorname{Sym}_{2}(V)$ has a natural structure of a Real vector space; cf. Remark 2.1 It follows that the image of $\mathbb{P}(V)$ under the Veronese embedding $\nu_{2}: \mathbb{P}(V) \hookrightarrow \mathbb{P}\left(\operatorname{Sym}_{2}(V)\right)$ becomes a Real subvariety of $\mathbb{P}\left(\operatorname{Sym}_{2}(V)\right)$. Define

$$
Q(V):=\mathrm{T}\left(\mathcal{O}_{\mathbb{P}(V)}(2)\right) .
$$

It is clear that $Q(V)$ can be identified with the complex suspension

$$
\mathbb{Z}_{\mathbb{C}}\left(\nu_{2}(\mathbb{P}(V))\right)=\nu_{2}(\mathbb{P}(V)) \# p_{\infty} \subset \mathbb{P}\left(\operatorname{Sym}_{2}(V) \oplus \mathbb{C}\right),
$$

where $p_{\infty}=\mathbb{P}(0 \oplus \mathbb{C}) \in \mathbb{P}\left(\operatorname{Sym}_{2}(V) \oplus \mathbb{C}\right)$. Let $\mathcal{Q}(V)=Q(V)-\left\{p_{\infty}\right\}$ denote the total space of $\mathcal{O}_{\mathbb{P}(V)}(2)$ and consider the Real bundles $\widetilde{\pi}, \widetilde{p}$ in the following pull-back diagram:

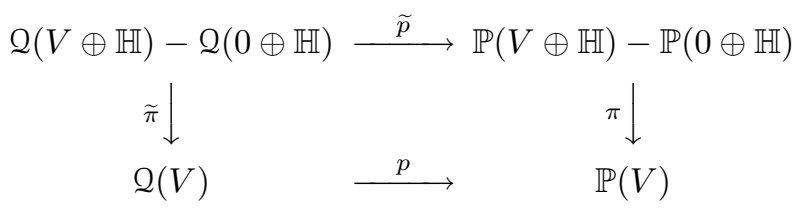


Applying the homotopy property for Real vector bundles dS03a, Prop. 4.15] to $\widetilde{\pi}$ and $\widetilde{p}$ in the diagram above, we obtain the following equivariant homotopy equivalences:

$$
\begin{aligned}
z_{1}(\mathbb{P}(V \oplus \mathbb{H})-\mathbb{P}(0 \oplus \mathbb{H})) & \stackrel{\tilde{p}^{*}}{\cong} z_{2}(Q(V \oplus \mathbb{H})-Q(0 \oplus \mathbb{H})) \\
& \stackrel{\left(\tilde{\pi}^{*}\right)^{-1}}{\cong} z_{0}(Q(V)) .
\end{aligned}
$$

It now follows that

$$
\begin{aligned}
z_{1}(\mathbb{P}(V \oplus \mathbb{H})) & \cong z_{1}(\mathbb{P}(\mathbb{H})) \times z_{1}(\mathbb{P}(V \oplus \mathbb{H})-\mathbb{P}(0 \oplus \mathbb{H})) \\
& \cong \mathbb{Z} \times z_{0}(\mathcal{Q}(V)) \\
& \cong z_{0}\left(\left\{p_{\infty}\right\}\right) \times z_{0}(\mathcal{Q}(V)) \\
& \cong z_{0}(Q(V)) .
\end{aligned}
$$

The preceding observations imply the following.

Theorem 3.4. For $k<n$ there are canonical equivariant homotopy equivalences:

$$
z^{2 k-1}\left(\mathbb{P}\left(\mathbb{H}^{n}\right)\right) \cong \prod_{j=1}^{k} F\left(\mathbb{P}(\mathbb{H})_{+}, K(\mathbb{Z}(2 j-1), 4 j-2)\right)
$$

and

$$
z^{2 k}\left(\mathbb{P}\left(\mathbb{H}^{n}\right)\right) \cong \prod_{j=0}^{k} F\left(\mathbb{P}(\mathbb{H})_{+}, K(\mathbb{Z}(2 j), 4 j)\right)
$$

Proof. The first equivalence follows from $z^{2 k-1}\left(\mathbb{P}\left(\mathbb{H}^{n}\right)\right) \cong z_{0}\left(\mathbb{P}\left(\mathbb{H}^{k}\right)\right)$ given by (26) and Corollary 2.4 To prove the second one, first consider the equivalences

$$
z^{2 k}\left(\mathbb{P}\left(\mathbb{H}^{n}\right)\right) \underset{[27}{\cong} z^{2 k}\left(\mathbb{P}\left(\mathbb{H}^{k+1}\right)\right)=z_{1}\left(\mathbb{P}\left(\mathbb{H}^{k+1}\right)\right) \underset{[29}{\cong} z_{0}\left(Q\left(\mathbb{H}^{k}\right)\right)
$$

and

$$
z_{0}\left(Q\left(\mathbb{H}^{k}\right)\right)=z_{0}\left(\mathrm{~T}\left(\mathcal{O}_{\mathbb{P}_{\left(\mathbb{H}^{k}\right)}}(2)\right)\right) \cong z_{0}\left(S^{2,1} \wedge \mathbb{P}\left(\mathbb{H}^{k}\right)_{+}\right) ;
$$

cf. (26), (27), (29) and Propositions 1.13 and 1.14 .

Using Corollary 2.3 one obtains

$$
\begin{aligned}
z_{0}\left(S^{2,1} \wedge \mathbb{P}\left(\mathbb{H}^{k}\right)_{+}\right)_{\mathrm{o}} & \cong \prod_{j=1}^{n} z_{0}\left(S^{2,1} \wedge\left\{\mathbb{P}\left(\mathbb{H}^{j}\right) / \mathbb{P}\left(\mathbb{H}^{j-1}\right)\right\}\right)_{\mathrm{o}} \\
& =\prod_{j=1}^{n} z_{0}\left(S^{2,1} \wedge\left\{\mathrm{T}\left(\mathcal{O}_{\mathbb{P}(\mathbb{H})}(1) \otimes \mathbb{H}^{j-1}\right)\right\}\right)_{\mathrm{o}} \\
& =\prod_{j=1}^{n} z_{0}\left(\mathrm{~T}\left(\left\{\mathcal{O}_{\mathbb{P}(\mathbb{H})}(1) \otimes \mathbb{H}^{j-1}\right\} \oplus \mathbf{1}\right)\right)_{\mathrm{o}}
\end{aligned}
$$

Finally, the canonical equivalence

$$
z_{0}\left(\mathrm{~T}\left(\left\{\mathcal{O}_{\mathbb{P}(\mathbb{H})}(1) \otimes \mathbb{H}^{j-1}\right\} \oplus \mathbf{1}\right)\right)_{\mathrm{o}} \cong F\left(\mathbb{P}(\mathbb{H})_{+}, z_{0}\left(S^{4 j, 2 j}\right)_{\mathrm{o}}\right)
$$

established in Propositions 1.13 and 1.14, along with (30), (31) and (32), proves the second assertion of the proposition. 
3.2. Stabilizations of cycle spaces. Here we use the group of algebraic cycles, with the quaternionic $\mathbb{Z} / 2$-action, to construct equivariant infinite loop spaces using the formalism of $(\mathbb{Z} / 2) \mathcal{J}_{*}$-functors explained in Appendix $\mathrm{A}$.

Consider a Real vector space $(V, \sigma)$ of complex dimension $v$, and let $V^{*}$ be its complex dual. Denote by $\left(V_{\mathbb{H}}, \sigma\right)$ the quaternionic vector space $V_{\mathbb{H}}:=V \otimes_{\mathbb{C}} \mathbb{H}$ with quaternionic structure $\mathbf{j}$ defined by $\mathbf{j}(\mathbf{v} \otimes h)=\sigma(\mathbf{v}) \otimes$ jh. If $V \neq\{0\}$, define

$$
z(V):=\coprod_{j=-2 v}^{2 v} z^{2 v+j}\left(V_{\mathbb{H}}^{*} \oplus V_{\mathbb{H}}\right)_{1},
$$

where $Z^{2 v+j}\left(\mathbb{P}\left(V_{\mathbb{H}}^{*} \oplus V_{\mathbb{H}}\right)\right)_{1}$ denotes the space of algebraic cycles of codimension $2 v+j$ and degree 1 in $\mathbb{P}\left(V_{\mathbb{H}}^{*} \oplus V_{\mathbb{H}}\right)$. Define $Z(\{0\})$ to be the one-point set $\left\{\mathbf{1}_{0}\right\}$. The involution induced by $\sigma$ on $\mathbb{P}\left(V_{\mathbb{H}}^{*} \oplus V_{\mathbb{H}}\right)$ gives $\mathcal{Z}(V)$ the structure of a Real space, with a natural basepoint $\mathbf{1}_{V}:=\mathbb{P}\left(V_{\mathbb{H}}^{*} \oplus\{0\}\right) \in \mathcal{Z}^{2 v}\left(V_{\mathbb{H}}^{*} \oplus V_{\mathbb{H}}\right)_{1} \subset \mathcal{Z}(V)$ which is fixed under the $\mathbb{Z} / 2$-action.

Let $(V, \sigma)$ and $\left(W, \sigma^{\prime}\right)$ be Real vector spaces of dimensions $v$ and $w$, respectively. Given an injective complex linear map $i: V \hookrightarrow W$ one has an induced map $(i d \oplus i)_{*}$ : $z^{2 v+j}\left(\mathbb{P}\left(V_{\mathbb{H}}^{*} \oplus V_{\mathbb{H}}\right)\right)_{1} \rightarrow z^{2 w+j}\left(\mathbb{P}\left(V_{\mathbb{H}}^{*} \oplus W_{\mathbb{H}}\right)\right)_{1}$, which is simply an inclusion map. Similarly, if $i^{*}: W^{*} \rightarrow V^{*}$ is the adjoint surjection, one has an induced map $\left(i^{*} \oplus i d\right)^{*}: z^{2 w+j}\left(\mathbb{P}\left(V_{\mathbb{H}}^{*} \oplus W_{\mathbb{H}}\right)\right)_{1} \rightarrow z^{2 w+j}\left(\mathbb{P}\left(W_{\mathbb{H}}^{*} \oplus W_{\mathbb{H}}\right)\right)_{1}$, given by the appropriate pull-back of cycles; cf. Remark 3.3. Note that these maps are closed topological embeddings. Define

$i_{\sharp}: z(V)=\coprod_{j=-2 v}^{2 v} z^{2 v+j}\left(\mathbb{P}\left(V_{\mathbb{H}}^{*} \oplus V_{\mathbb{H}}\right)\right)_{1} \longrightarrow z(W)=\coprod_{j=-2 w}^{2 w} z^{2 w+j}\left(\mathbb{P}\left(W_{\mathbb{H}}^{*} \oplus W_{\mathbb{H}}\right)\right)_{1}$

as the composition $\left(i^{*} \oplus i d\right)^{*} \circ(i d \oplus i)_{*}$.

Following the notation in Appendix $\mathbb{A}$ we use $\operatorname{Mor}_{(\mathbb{Z} / 2) \mathcal{J}_{*}}(V, W)$ to denote the space of complex linear embeddings from $V$ to $W$, where $(V, \sigma)$ and $(W, \tau)$ are finite dimensional Real vector spaces.

Lemma 3.5. Let $(V, \sigma)$ and $(W, \tau)$ be Real vector spaces. The assignment $i \mapsto i_{\sharp}$ defines $a(\mathbb{Z} / 2)$-map $Z: \operatorname{Mor}_{(\mathbb{Z} / 2) \mathcal{J}_{*}}(V, W) \rightarrow \mathcal{M} \mathfrak{M} \mathfrak{p}(Z(V), Z(W))$, where $\mathbb{Z} / 2$ acts on both $\operatorname{Mor}_{(\mathbb{Z} / 2) \mathcal{J}_{*}}(V, W)$ and $\mathcal{M} \mathfrak{M p}(Z(V), Z(W))$ via conjugation.

It follows that $V \mapsto z(V)$ defines a functor from the category $\mathbb{R J}_{*}$ of Real vector spaces and complex linear embeddings to the category $\mathcal{T}_{\mathbb{Z} / 2}$ of Real spaces and arbitrary continuous maps. Furthermore, the statement in Lemma 3.5 expresses precisely the fact that this functor is enriched over the category $(\mathbb{Z} / 2) \mathcal{T}$ of Real spaces and equivariant maps; cf. Appendix A.1.

We now show that $z(-)$ comes with an additional structure, an equivariant "Whitney sum" pairing, defined as follows. Given real vector spaces $(V, \sigma)$ and $\left(W, \sigma^{\prime}\right)$, define

$$
\#_{V, W}: z(V) \times z(W) \rightarrow z(V \oplus W)
$$


as the map whose restrictions to the components of $z(V)$ are given by the compositions

$$
\begin{aligned}
z^{2 v+j}\left(\mathbb{P}\left(V_{\mathbb{H}}^{*} \oplus V_{\mathbb{H}}\right)\right)_{1} \times z^{2 w+k}\left(\mathbb{P}\left(W_{\mathbb{H}}^{*} \oplus W_{\mathbb{H}}\right)\right)_{1} \\
\stackrel{\#}{\longrightarrow} z^{2(w+v)+j+k}\left(\mathbb{P}\left(V_{\mathbb{H}}^{*} \oplus V_{\mathbb{H}} \oplus W_{\mathbb{H}}^{*} \oplus W_{\mathbb{H}}\right)\right)_{1} \\
\stackrel{\tau}{\rightarrow} z^{2(w+v)+j+k}\left(\mathbb{P}\left((V \oplus W)_{\mathbb{H}}^{*} \oplus(V \oplus W)_{\mathbb{H}}\right)\right)_{1},
\end{aligned}
$$

where \# is the join pairing (37). Here $\tau$ denotes the map on cycles induced by the composition of linear isomorphisms $V_{\mathbb{H}}^{*} \oplus V_{\mathbb{H}} \oplus W_{\mathbb{H}}^{*} \oplus W_{\mathbb{H}} \rightarrow\left(V_{\mathbb{H}}^{*} \oplus W_{\mathbb{H}}^{*}\right) \oplus\left(V_{\mathbb{H}} \oplus W_{\mathbb{H}}\right) \rightarrow$ $(V \oplus W)_{\mathbb{H}}^{*} \oplus(V \oplus W)_{\mathbb{H}}$, where the former map is a shuffle isomorphism and the latter is the usual natural identification. Define $\#_{0, W}$ to be the identity map, after the natural identification $\mathcal{Z}(\mathbb{P}(0 \oplus V)) \equiv \mathcal{Z}(\mathbb{P}(V))$.

Proposition 3.6. The assignments $V \mapsto Z(V)$ along with the pairings $\#_{V, W}$ give $\mathcal{Z}(-)$ the structure of an equivariant Real $(\mathbb{Z} / 2) J^{*}$-functor; cf. Definition $A .8$ in Appendix A

Proof. Condition (i) in Definition A.2 follows from the definition of $\#_{V, W}$, and condition (iii) is precisely the content of Lemma 3.5. The fact that $Z(-)$ is a continuous covariant functor $\mathcal{Z}:(\mathbb{Z} / 2) \mathcal{J}_{*} \rightarrow \mathcal{T}_{\mathbb{Z} / 2}$ and that \# gives a (coherently) commutative, associative and continuous natural transformation $\#: z \times z \rightarrow z$ follows exactly as in the non-equivariant case; cf. BLLF93. We prove in (24) that $\#_{V, W}$ is a $(\mathbb{Z} / 2)$-map, and this gives the last condition in Definition A.8

Consider our preferred complete $\mathbb{Z} / 2$-universe $\left(\mathbb{C}^{\infty}, \sigma\right)$, where $\sigma$ is complex conjugation; cf. Appendix A. Following (67) define

$$
z_{\mathbb{H}}:=\underset{V \subset \lim ^{\infty}}{\longrightarrow} z(V)
$$

where $V$ runs over all Real subspaces of $\left(\mathbb{C}^{\infty}, \sigma\right)$. Since the increasing coordinate flag $\{0\} \subset \mathbb{C} \subset \mathbb{C}^{2} \subset \cdots \subset \mathbb{C}^{\infty}$ is cofinal among the finite dimensional subspaces of $\mathbb{C}^{\infty}$, one observes that

$$
z_{\mathbb{H}}=\underset{n}{\lim } z\left(\mathbb{C}^{n}\right)=\lim _{n} \coprod_{j=-2 n}^{2 n} z^{2 n+j}\left(\mathbb{P}\left(\mathbb{H}^{n *} \oplus \mathbb{H}^{n}\right)\right)_{1} .
$$

Here we are making the usual identification $\mathbb{C}_{\mathbb{H}}^{n}=\mathbb{C}^{n} \otimes_{\mathbb{C}} \mathbb{H} \equiv \mathbb{H}^{n}$. The maps $i_{\sharp}$ in the colimit above are compositions of algebraic suspensions and coordinatewise inclusions. Therefore, they are compatible with the splittings given in Theorem 3.4.

Theorem 3.7. The space $\mathcal{Z}_{\mathbb{H}}$ is written as a disjoint union of connected spaces

$$
z_{\mathbb{H}}=\coprod_{j=-\infty}^{\infty} z_{\mathbb{H}}^{j}
$$

where the equivariant homotopy type of $Z_{\mathbb{H}}^{j}$ is totally determined by

$$
z_{\mathbb{H}}^{j} \cong \begin{cases}\left(\prod_{j=1}^{\infty} F\left(\mathbb{P}(\mathbb{H})_{+}, K(\mathbb{Z}(2 j-1), 4 j-2)\right)\right)_{1} & \text { if } j \text { is odd, } \\ \prod_{j=1}^{\infty} F\left(\mathbb{P}(\mathbb{H})_{+}, K(\mathbb{Z}(2 j), 4 j)\right) & \text { if } j \text { is even, }\end{cases}
$$

where the subscript 1 denotes the component of the non-equivariant fundamental class of $\mathbb{P}(\mathbb{H})$. 
Proof. It is evident that if one defines

$$
Z_{\mathbb{H}}^{j}:=\underset{n}{\lim _{\longrightarrow}} Z^{j+2 n}\left(\mathbb{P}\left(\mathbb{H}^{n *} \oplus \mathbb{H}^{n}\right)\right)_{1},
$$

then $z_{\mathbb{H}}=\coprod_{j=-\infty}^{\infty} z_{\mathbb{H}}^{j}$. The result now follows from the remark preceding the theorem together with Theorem 3.4 .

Remark 3.8. Note the the equivariant homotopy type of $z_{\mathbb{H}}^{j}$ is completely determined by the parity of $j$. Furthermore, a canonical inclusion of coordinate hyperplanes gives immediately an equivariant homotopy equivalence $z_{\mathbb{H}}^{j} \cong z_{\mathbb{H}}^{j+2}$, for all $j$. For this reason we establish the notation

$$
z_{\mathbb{H}}^{\mathrm{ev}}:=\text { the equivariant homotopy type of } z_{\mathbb{H}}^{j} \text {, for } j \text { even, }
$$

and

$$
z_{\mathbb{H}}^{\text {odd }}:=\text { the equivariant homotopy type of } z_{\mathbb{H}}^{j} \text {, for } j \text { odd. }
$$

The spaces $z_{\mathbb{H}}^{\mathrm{ev}}$ and $z_{\mathbb{H}}^{\text {odd }}$ are both connected. Also, it is easy to see that the fixed point set $\left(\mathcal{Z}_{\mathbb{H}}^{\text {odd }}\right)^{\mathbb{Z} / 2}$ is empty while $\left(\mathcal{Z}_{\mathbb{H}}^{e v}\right)^{\mathbb{Z} / 2}$ is connected; see [LLFM98a]. Since the join operation is additive on the codimension of cycles it induces group structures on the set of connected components of $z_{\mathbb{H}}$ and $z_{\mathbb{H}}^{\mathbb{Z} / 2}$ satisfying

$$
\pi_{0}\left(z_{\mathbb{H}}^{\mathbb{Z} / 2}\right) \cong 2 \mathbb{Z} \subset \mathbb{Z} \cong \pi_{0}\left(\mathcal{Z}_{\mathbb{H}}\right) .
$$

We now explore the fact that $z_{\mathbb{H}}=\lim _{\backslash \subset \mathcal{U}} z(V)$ for the $\mathbb{R J}_{*}$-functor $z$. First observe that $\mathcal{Z}_{\mathbb{H}}$ has an action of the equivariant linear isometries operad $\mathcal{L}_{*}(\mathcal{U})$, according to Proposition A.9. Observe that the $H$-space structure on both $Z_{\mathbb{H}}$ and its fixed point set $\left(\mathcal{Z}_{\mathbb{H}}\right)^{\mathbb{Z} / 2}$ is induced by the join operation. Hence, it follows from Remark 3.8 that $\mathcal{Z}_{\mathbb{H}}$ is $\mathbb{Z} / 2$-group-like; cf. Definition A.6. This fact and Theorem A.7 yield our next result.

Theorem 3.9. The join operation makes the space $\mathbb{Z}_{\mathbb{H}}$ equivariantly homotopy equivalent to an equivariant $\mathbb{Z} / 2$-infinite loop space.

Definition 3.10. The infinite loop space structure on $z_{\mathbb{H}}$ determines an equivariant spectrum (cf. May96) denoted $\mathfrak{Z}_{\mathbb{H}}$, satisfying $\mathfrak{Z}_{\mathbb{H}}(0) \cong \mathcal{Z}_{\mathbb{H}}$.

\section{Quaternionic $K$-THEORY}

In LLFM98b] the connective version of $K R$-theory is studied from the equivariant point of view, along with a suitable theory of equivariant Chern classes. In this section and the next, we provide quaternionic analogues, along with a suitable theory of equivariant Chern classes and their equivariant deloopings. We must point out that in [Dup99], Dupont conjectures the existence of an appropriate theory of Chern classes for quaternionic bundles. Here we provide a natural answer to his question.

4.1. Classifying spaces and equivariant quaternionic $K$-theory spectrum. Given a Real vector space $(V, \sigma)$ of complex dimension $v$, we follow (35) and define

$$
\operatorname{Gr}(V):=\coprod_{j=-2 v}^{2 v} \operatorname{Gr}^{2 v+j}\left(V_{\mathbb{H}}^{*} \oplus V_{\mathbb{H}}\right) .
$$


The assignment $V \mapsto \operatorname{Gr}(V)$ becomes functorial, as follows. Consider an inclusion $i$ : $W \hookrightarrow V$ of Real vector spaces of dimensions $w$ and $v$, respectively. As in the previous section, we observe that $i$ induces an inclusion $(i d \oplus i)_{*}: \mathrm{Gr}^{2 w+j}\left(W_{\mathbb{H}}^{*} \oplus W_{\mathbb{H}}\right) \rightarrow$ $\mathrm{Gr}^{2 v+j}\left(W_{\mathbb{H}}^{*} \oplus V_{\mathbb{H}}\right)$ given by the inclusion of linear subspaces, and that the surjection $i^{*}: V^{*} \rightarrow W^{*}$ induces another inclusion $\left(i^{*} \oplus i d\right)^{-1}: \mathrm{Gr}^{2 v+j}\left(W_{\mathbb{H}}^{*} \oplus V_{\mathbb{H}}\right) \rightarrow$ $\operatorname{Gr}^{2 v+j}\left(V_{\mathbb{H}}^{*} \oplus V_{\mathbb{H}}\right)$, given by taking inverse images. Let $i_{\sharp}: \operatorname{Gr}(W) \rightarrow \operatorname{Gr}(V)$ be the map given, on each connected component, by the composition

$$
i_{\sharp}:=\left(i^{*} \oplus i d\right)^{-1} \circ(i d \oplus i)_{*} .
$$

Given a Real space $X$, and any Real vector space $(V, \sigma)$, we denote by $\underline{V}_{\mathbb{H}}$ the quaternionic vector bundle $X \times V_{\mathbb{H}}$ over $X$. Now, let $\xi_{V}^{j}$ denote the universal quotient bundle over $\mathrm{Gr}^{2 v+j}\left(V_{\mathbb{H}}^{*} \oplus V_{\mathbb{H}}\right)$. The proof of the following result is standard.

Lemma 4.1. The map on Grassmannians

$$
i_{\sharp}: G r^{2 w+j}\left(W_{\mathbb{H}}^{*} \oplus W_{\mathbb{H}}\right) \rightarrow G r^{2 v+j}\left(V_{\mathbb{H}}^{*} \oplus V_{\mathbb{H}}\right),
$$

induced by an inclusion $i: W \hookrightarrow V$, satisfies

$$
\left.\left(i_{\sharp}\right)^{*} \xi_{V}^{j}=\xi_{W}^{j} \oplus \underline{(V / W}\right)_{\mathbb{H}} .
$$

Definition 4.2. Define the Real space $(\mathbb{Z} \times B U)_{\mathbb{H}}$ as the colimit

$$
(\mathbb{Z} \times B U)_{\mathbb{H}}:=\lim _{V \overrightarrow{\subset \mathbb{C}^{\infty}}} \operatorname{Gr}(V) .
$$

Observe that, in the same fashion as $\mathcal{Z}_{\mathbb{H}}\left(\mathrm{cf}\right.$. Theorem 3.7), the space $(\mathbb{Z} \times B U)_{\mathbb{H}}$ can be written as a disjoint union $\coprod_{j=-\infty}^{\infty} \mathrm{BU}_{\mathbb{H}}^{j}$ of Real spaces $\mathrm{BU}_{\mathbb{H}}^{j}$ defined as $\mathrm{BU}_{\mathbb{H}}^{j}:=\underline{\lim }_{V \subset \mathbb{C}^{\infty}} \mathrm{Gr}^{2 v+j}\left(V_{\mathbb{H}}^{*} \oplus V_{\mathbb{H}}\right)$. Furthermore, the coordinatewise inclusion $\mathbb{C}^{\infty} \hookrightarrow \mathbb{C}^{\infty}$, given by setting the first coordinate to zero, induces equivariant homotopy equivalences

$$
\mathrm{BU}_{\mathbb{H}}^{j} \cong \mathrm{BU}_{\mathbb{H}}^{j+2} \cong \cdots \cong \mathrm{BU}_{\mathbb{H}}^{j+2 r} \cong \cdots
$$

Hence all connected components of $(\mathbb{Z} \times B U)_{\mathbb{H}}$ are either equivalent to $\mathrm{BU}_{\mathbb{H}}^{0}$ or to $\mathrm{BU}_{\mathbb{H}}^{1}$, and we denote by $\mathrm{BU}_{\mathbb{H}}^{e v}$ and $\mathrm{BU}_{\mathbb{H}}^{\text {odd }}$ their respective equivariant homotopy types.

Proposition 4.3. The space $(\mathbb{Z} \times B U)_{\mathbb{H}}$ classifies quaternionic $K$-theory. In other words, given a compact Real space $X$ one has a natural isomorphism

$$
\left[X_{+},(\mathbb{Z} \times B U)_{\mathbb{H}}\right]_{\mathbb{Z} / 2} \stackrel{\simeq}{\rightrightarrows} K H^{0}(X) .
$$

Proof. The proof follows standard arguments, as in Seg68, and we only outline the details which are particular to this case. Let $(X, \sigma)$ be a compact Real space. Given an equivariant map $f: X \rightarrow(\mathbb{Z} \times B U)_{\mathbb{H}}$, one can find a Real subspace $W \subset \mathbb{C}^{\infty}$, $\operatorname{dim} W=w$, so that the map $f$ factors as a composition

$$
X \stackrel{f_{W}}{\longrightarrow} \operatorname{Gr}(W) \stackrel{i_{W}}{\longrightarrow} \coprod_{j=-2 w}^{2 w} \mathrm{BU}_{\mathbb{H}}^{j} \subset(\mathbb{Z} \times B U)_{\mathbb{H}},
$$

where the $i_{W}$ 's are the natural maps from the directed system defining $(\mathbb{Z} \times B U)_{\mathbb{H}}$.

Now, assign to $f$ the isomorphism class of the virtual bundle $f_{W}^{*}\left(\xi_{W}\right)-\underline{W}_{\mathbb{H}}$, where $\xi_{W}$ is the bundle over $\operatorname{Gr}(W)$ whose restriction to the component $\operatorname{Gr}^{2 w+j}\left(W_{\mathbb{H}}^{*} \oplus W_{\mathbb{H}}\right)$ is the universal quotient bundle $\xi_{W}^{j}$. If $i: W \hookrightarrow V \subset \mathbb{C}^{\infty}$ 
is an inclusion, then it follows from the construction of $\mathrm{BU}_{\mathbb{H}}^{j}$ that $f_{V}=i_{\sharp} \circ f_{W}$, and hence one has equalities in $K H^{0}(X)$ :

$$
\begin{aligned}
f_{V}^{*}\left(\xi_{V}\right)-\underline{V}_{\mathbb{H}} & =f_{W}^{*}\left(i_{\mathbb{H}}^{*}\left(\xi_{V}\right)\right)-\underline{V}_{\mathbb{H}}=\left(f_{W}^{*}\left(\xi_{W}\right) \oplus \underline{(V / W)_{\mathbb{H}}}\right)-\underline{V}_{\mathbb{H}} \\
& =f_{W}^{*}\left(\xi_{W}\right)+\underline{(V / W)_{\mathbb{H}}}-\underline{V}_{\mathbb{H}}=f_{W}^{*}\left(\xi_{W}\right)-\underline{W}_{\mathbb{H}},
\end{aligned}
$$

where the second one follows from Lemma 4.1 This shows that the element in $K H^{0}(X)$ thus obtained is independent of the factorization through a finite dimensional Grassmannian. Standard arguments Seg68 show that this assignment only depends on the equivariant homotopy class of $f$ and that the resulting map $\Psi:\left[X_{+},(\mathbb{Z} \times B U)_{\mathbb{H}}\right]_{\mathbb{Z} / 2} \rightarrow K H^{0}(X)$ is injective.

In order to prove surjectivity, let $(E, \tau)$ be a quaternionic bundle over $(X, \sigma)$. One can find sections $s_{i}: X \rightarrow E, i=1, \ldots, k$ generating $E$ and define $\phi: \mathbb{H}^{n} \times X \rightarrow E$ by sending $\left(a_{1}+b_{1} j, \ldots, a_{k}+b_{k} j ; x\right)$ to $\sum_{i=1}^{k} a_{i} s_{i}(x)+\sum_{i=1}^{k} b_{i} \tau\left(s_{i}(\sigma x)\right) \in E_{x}$. It is clear that $\phi$ is onto and that $\phi$ is a map of quaternionic bundles for the diagonal quaternionic structure on $\mathbb{H}^{n} \times X$ given by left multiplication by $\jmath$ on the first factor, and by $\sigma$ on the second factor. It follows that the map $f: X \rightarrow \operatorname{Gr}^{\operatorname{dim} E}\left(\mathbb{H}^{k}\right)$ defined as $f(x)=\operatorname{ker}\left(\phi_{x}\right)$ is equivariant and satisfies $f^{*} Q \cong E$, where $Q$ is the universal quotient bundle over $\operatorname{Gr}^{\operatorname{dim} E}\left(\mathbb{H}^{k}\right)$. The surjectivity of $\Psi$ follows.

Definition 4.4. For each $j \in \mathbb{Z}$, we denote by $\xi^{j}$ the virtual universal quotient bundle over $\mathrm{BU}_{\mathbb{H}}^{j}$, of virtual dimension $j$, whose restriction to $\operatorname{Gr}^{2 v+j}\left(V_{\mathbb{H}}^{*} \oplus V_{\mathbb{H}}\right)$ is $\xi_{V}^{j}-V_{\mathbb{H}}$.

Notice that the construction of $\operatorname{Gr}(V)$ here parallels that of $z(V)$, given in (35). Furthermore, given Real vector spaces $(V, \sigma),\left(W, \sigma^{\prime}\right)$ one can define

$$
\oplus_{V, W}: \operatorname{Gr}(V) \times \operatorname{Gr}(W) \rightarrow \operatorname{Gr}(V \oplus W)
$$

by sending $L \subset V_{\mathbb{H}}^{*} \oplus V_{\mathbb{H}}$ and $L^{\prime} \subset W_{\mathbb{H}}^{*} \oplus W_{\mathbb{H}}$ to $\tau(L \oplus L)$, where $\tau$ is the shuffle map which switches coordinates from $\left(V_{\mathbb{H}}^{*} \oplus V_{\mathbb{H}}\right) \oplus\left(W_{\mathbb{H}}^{*} \oplus W_{\mathbb{H}}\right)$ to $\left(V_{\mathbb{H}} \oplus W_{\mathbb{H}}\right)^{*} \oplus\left(V_{\mathbb{H}} \oplus W_{\mathbb{H}}\right)$. The following proposition is analogous to Proposition 3.6 and is proven in a similar fashion.

Proposition 4.5. The assignments $V \mapsto \operatorname{Gr}(V)$ along with the pairings $\oplus_{V, W}$ give $\operatorname{Gr}(-)$ the structure of an equivariant $(\mathbb{Z} / 2) \mathcal{J}^{*}$-functor, in the language of [May77]. See also [LLFM96].

The proof of the following result is identical to the proof of Theorem 3.9.

Theorem 4.6. The direct sum operation induces an equivariant $\mathbb{Z} / 2$-infinite loop space structure on the space $(\mathbb{Z} \times B U)_{\mathbb{H}}$.

Definition 4.7. The infinite loop space structure on $(\mathbb{Z} \times B U)_{\mathbb{H}}$ determines an equivariant spectrum (cf. May96) denoted $\mathfrak{K} \mathfrak{s p}$, satisfying $\mathfrak{K} \mathfrak{s p}(0) \cong(\mathbb{Z} \times B U)_{\mathbb{H}}$. This is the connective quaternionic K-theory spectrum.

Remark 4.8. The same construction with Real vector spaces instead of quaternionic ones would give $(\mathbb{Z} \times B U)_{\mathbb{R}}$ along with the equivariant infinite loop space structure classifying $K R$-theory.

Let $(V, \sigma)$ be a Real vector space. An important feature of our constructions is the fact that a complex linear subspace $L$ of codimension $2 v+j$ in $V_{\mathbb{H}}^{*} \oplus V_{\mathbb{H}}$ is also an irreducible subvariety of codimension $2 v+j$ and degree 1 in 
$\mathbb{P}\left(V_{\mathbb{H}}^{*} \oplus V_{\mathbb{H}}\right)$. Furthermore, the external direct sum of two such subspaces corresponds to their algebraic join when seen as projective subvarieties. See [BLLF93] for more details. This gives a natural transformation $c: \mathcal{Z}(-) \rightarrow \operatorname{Gr}(-)$ of $(\mathbb{Z} / 2) \mathcal{J}_{*^{-}}$ functors. Standard arguments, such as in BLLF93, show that the resulting map of colimits

$$
c^{\mathbb{H}}:(\mathbb{Z} \times B U)_{\mathbb{H}} \rightarrow z_{\mathbb{H}}
$$

is a map of equivariant infinite loop spaces. In other words, it induces a map of equivariant spectra $c^{\mathbb{H}}: \mathfrak{K}_{\mathfrak{s p}} \rightarrow \mathfrak{Z}_{\mathbb{H}}$.

Definition 4.9. The map $c^{\mathbb{H}}: \mathfrak{K}_{\mathfrak{s p}} \rightarrow \mathfrak{Z}_{\mathbb{H}}$ is called the total quaternionic Chern class map.

In order to analyze the quaternionic Chern class map in the level of classifying spaces, we first need to understand the equivariant cohomology of $(\mathbb{Z} \times B U)_{\mathbb{H}} \times \mathbb{P}(\mathbb{H})$. This computation and the subsequent analysis form the content of our next section.

\section{Characteristic Classes}

In this section we introduce characteristic classes for quaternionic bundles and establish their relation to the total quaternionic Chern class map $c^{\mathbb{H}}:(\mathbb{Z} \times B U)_{\mathbb{H}} \rightarrow$ $z_{\mathbb{H}}$, in the level of classifying spaces.

Definition 5.1. Let $E$ be a rank $e$ quaternionic bundle over a Real space $X$. For $\delta=0,1$, and $i$ satisfying $0 \leq 2 i+\delta \leq e$, define the quaternionic Chern classes of $E$ by

$$
c_{e-(2 i+\delta)}^{\mathbb{H I}}(E):=c_{e-(2 i+\delta)}^{\mathbb{R}}(E \otimes \mathcal{O}(1))-(i+\delta) c_{e-(2 i+\delta)-1}^{\mathbb{R}}(E \otimes \mathcal{O}(1)) \mathbf{x},
$$

where $c_{-1}(-)=0$ and $\mathbf{x}$ is defined in Remark 1.19

We first compute the equivariant cohomology of $(\mathbb{Z} \times B U)_{\mathbb{H}} \times \mathbb{P}(\mathbb{H})$ and then establish its relation to the total Chern class map $c^{\mathbb{H}}$.

5.1. Cohomology of $(\mathbb{Z} \times B U)_{\mathbb{H}}$. Observe that all components of $(\mathbb{Z} \times B U)_{\mathbb{H}} \times \mathbb{P}(\mathbb{H})$ have the same equivariant homotopy type, hence it suffices to compute $H^{*, *}\left(\mathrm{BU}_{\mathbb{H}}^{e v} \times \mathbb{P}(\mathbb{H}) ; \underline{Z}\right)$. An explicit equivariant homotopy equivalence $\Psi: \mathrm{BU}_{\mathbb{H}}^{e v} \times$ $\mathbb{P}(\mathbb{H}) \rightarrow \mathrm{BU}_{\mathbb{H}}^{\text {odd }} \times \mathbb{P}(\mathbb{H})$ can be constructed as follows. Given a Real vector space $(V, \sigma)$, let

$$
\psi_{V}^{j, j+1}: \operatorname{Gr}^{2 v+j}\left(V_{\mathbb{H}}^{*} \oplus V_{\mathbb{H}}\right) \times \mathbb{P}(\mathbb{H}) \rightarrow \mathrm{Gr}^{2 v+j+1}\left((V \oplus \mathbb{C})_{\mathbb{H}}^{*} \oplus(V \oplus \mathbb{C})_{\mathbb{H}}\right)
$$

denote the composition

$$
\begin{aligned}
\operatorname{Gr}^{2 v+j}\left(V_{\mathbb{H}}^{*} \oplus V_{\mathbb{H}}\right) \times \mathbb{P}(\mathbb{H}) \stackrel{i d \times \iota}{\longrightarrow} \operatorname{Gr}^{2 v+j}\left(V_{\mathbb{H}}^{*} \oplus V_{\mathbb{H}}\right) \times \operatorname{Gr}^{1}\left(\mathbb{H}^{*} \oplus \mathbb{H}\right) \\
\stackrel{\oplus_{V, \mathrm{c}}}{\longrightarrow} \mathrm{Gr}^{2 v+j+1}\left((V \oplus \mathbb{C})_{\mathbb{H}}^{*} \oplus(V \oplus \mathbb{C})_{\mathbb{H}}\right),
\end{aligned}
$$

where $\iota: \mathbb{P}(\mathbb{H}) \rightarrow \operatorname{Gr}^{1}\left(\mathbb{H}^{*} \oplus \mathbb{H}\right)$ is the linear embedding $L \hookrightarrow L \oplus \mathbb{H}$ and $\oplus_{V, \mathbb{C}}$ is the map (47).

It is easy to see that

$$
\left(\psi_{V}^{j, j+1}\right)^{*}\left(\xi_{V \oplus \mathbb{C}}^{j+1}\right)=\pi_{1}^{*}\left(\xi_{V}^{j}\right) \oplus \underline{\mathbb{H}} \oplus \pi_{2}^{*}(\mathcal{O}(1)),
$$

where the $\pi_{i}$ 's are the projections from $\mathrm{Gr}^{2 v+j}\left(V_{\mathbb{H}}^{*} \oplus V_{\mathbb{H}}\right) \times \mathbb{P}(\mathbb{H})$ to the respective factors, $\xi_{V}^{j}$ is the universal quotient bundle over the Grassmannian, and $\mathcal{O}(1)$ is the 
hyperplane bundle over $\mathbb{P}(\mathbb{H})$, which is a quaternionic bundle. The maps $\psi_{V}^{j, j+1}$ assemble to give a morphism of directed systems inducing a Real map $\psi^{j, j+1}$ : $\mathrm{BU}_{\mathbb{H}}^{j} \times \mathbb{P}(\mathbb{H}) \rightarrow \mathrm{BU}_{\mathbb{H}}^{j+1}$ with the property that $\left(\psi^{j, j+1}\right)^{*}\left(\xi^{j+1}\right)=\xi^{j} \times \mathcal{O}(1):=$ $\pi_{1}^{*}\left(\xi^{j}\right) \oplus \pi_{2}^{*}(\mathcal{O}(1))$.

We thus obtain a Real map $\psi: \mathrm{BU}_{\mathbb{H}}^{e v} \times \mathbb{P}(\mathbb{H}) \rightarrow \mathrm{BU}_{\mathbb{H}}^{\text {odd }}$ and define

$$
\Psi:=\psi \times \mathrm{id}: \mathrm{BU}_{\mathbb{H}}^{e v} \times \mathbb{P}(\mathbb{H}) \rightarrow \mathrm{BU}_{\mathbb{H}}^{\text {odd }} \times \mathbb{P}(\mathbb{H}) .
$$

Since $\Psi$ is a non-equivariant homotopy equivalence between free $(\mathbb{Z} / 2)$-spaces, one concludes that it is an equivariant homotopy equivalence.

Remark 5.2. The same procedure can be used to produce an equivariant homotopy equivalence $\Phi: z_{\mathbb{H}}^{\mathrm{ev}} \times \mathbb{P}(\mathbb{H}) \rightarrow z_{\mathbb{H}}^{\text {odd }} \times \mathbb{P}(\mathbb{H})$.

It is easy to see that the classes $c_{k}^{\mathbb{H}}\left(\xi_{W}^{j}\right)$ (see Lemma 4.1) are compatible with the directed system (44). Hence, they yield elements $\mathbf{c}_{k}^{\mathbb{H}} \in H^{2 k, k}\left(\mathrm{BU}_{\mathbb{H}}^{j} \times \mathbb{P}(\mathbb{H}) ; \underline{\mathbb{Z}}\right)$ that are compatible with the equivalences $\mathrm{BU}_{\mathbb{H}}^{j} \cong \mathrm{BU}_{\mathbb{H}}^{j+2}$, described in (46). It follows that we obtain well-defined classes in $H^{2 k, k}\left(\mathrm{BU}_{\mathbb{H}}^{e v} \times \mathbb{P}(\mathbb{H}) ; \underline{\mathbb{Z}}\right)$. Using the homotopy equivalence $\Psi$ we define corresponding classes in $\mathrm{BU}_{\mathbb{H}}^{e v} \times \mathbb{P}(\mathbb{H})$, and also denote these classes by $\mathbf{c}_{k}^{\mathbb{H}}$. The following facts are easily verified:

(a) Let $f: X \rightarrow(\mathbb{Z} \times B U)_{\mathbb{H}}$ be a classifying map for a quaternionic bundle $E$ over $X$. Then:

$$
c_{k}^{\mathbb{H}}(E):=(f \times \mathrm{id})^{*}\left(\mathbf{c}_{k}^{\mathbb{H}}\right) .
$$

(b) Let $E \rightarrow X$ be a quaternionic bundle of rank $e$, and let $\rho$ denote the forgetful functor from equivariant cohomology to singular cohomology. Then, denoting the fundamental cohomology class of $\mathbb{P}(\mathbb{H})$ by $\beta$, we have

$$
\rho\left(c_{k}^{\mathbb{H}}(E)\right)= \begin{cases}c_{k}(E) \times 1+c_{k-1}(E) \times \beta, & k \equiv e \bmod 2, \\ c_{k}(E) \times 1, & k \neq e \bmod 2 .\end{cases}
$$

In particular, considering the universal bundles $\xi_{k}^{j}$ over $\mathrm{Gr}^{2 k+j}\left(\mathbb{H}^{k^{*}} \oplus \mathbb{H}^{k}\right)$, we see that the classes $\rho\left(\mathbf{c}_{k}^{\mathbb{H}}\right)$ generate the singular cohomology of $(\mathbb{Z} \times B U)_{\mathbb{H}}$ $\times \mathbb{P}(\mathbb{H})$ over $H^{*}(\mathbb{P}(\mathbb{H}) ; \mathbb{Z})$.

Theorem 5.3. Let $\mathbf{c}_{k}^{\mathbb{H}}$ be the classes defined in (5.1). Then we have a ring isomorphism

$$
H^{*, *}\left(B U_{\mathbb{H}}^{e v} \times \mathbb{P}(\mathbb{H}) ; \underline{\mathbb{Z}}\right) \cong H^{*, *}(\mathbb{P}(\mathbb{H}) ; \underline{\mathbb{Z}})\left[\mathbf{c}_{1}^{\mathbb{H}}, \mathbf{c}_{2}^{\mathbb{H}}, \ldots, \mathbf{c}_{k}^{\mathbb{H}}, \ldots\right]
$$

Proof. Since $\mathrm{BU}_{\mathbb{H}}^{e v} \times \mathbb{P}(\mathbb{H})$ is free, it suffices to compute the Galois-Grothendieck cohomology groups with $\mathbb{Z}(n)$ coefficients. For this we use the spectral sequence (cf. Section 1.7)

$$
E_{2}^{r, s}(n):=H^{r}\left(B \mathbb{Z} / 2 ; \mathcal{H}\left(\mathrm{BU}_{\mathbb{H}}^{e v} \times \mathbb{P}(\mathbb{H}) ; \mathbb{Z}(n)\right)\right) \Rightarrow \hat{H}_{\mathbb{Z} / 2}^{r+s}\left(\mathrm{BU}_{\mathbb{H}}^{e v} \times \mathbb{P}(\mathbb{H}) ; \mathbb{Z}(n)\right),
$$

and the pairing of spectral sequences $E_{r}^{p, q}(n) \otimes E_{r}^{p^{\prime}, q^{\prime}}\left(n^{\prime}\right) \rightarrow E_{r}^{p+p^{\prime}, q+q^{\prime}}\left(n+n^{\prime}\right)$, induced by the cup product. This pairing makes $E_{r}^{*, *}(*)$ into a spectral sequence of $\mathbb{Z}^{2} \times \mathbb{Z} / 2$-graded rings.

Set $F_{2}^{p, q}(n):=H^{r}(B \mathbb{Z} / 2 ; \mathcal{H}(\mathbb{P}(\mathbb{H}) ; \mathbb{Z}(n)))$, and note that $E_{2}^{*, *}(*)$ is a module over $F_{2}^{*, *}(*)$. Corresponding to each of the classes $\mathbf{c}_{k}^{\mathbb{H}}$ are elements in $E_{2}^{0,2 k}(k)$ which are universal cycles. These cycles are denoted $\widetilde{d}_{k}$. The correspondence $\mathbf{c}_{k}^{\mathbb{H}} \mapsto \widetilde{d}_{k}$ defines a ring homomorphism $\Theta: F_{2}^{*, *}(*)\left[\mathbf{c}_{1}^{\mathbb{H}}, \mathbf{c}_{2}^{\mathbb{H}}, \ldots, \mathbf{c}_{n}^{\mathbb{H}}, \ldots\right] \rightarrow E_{2}^{*, *}(*)$. 
The quaternionic involution induces a map $\sigma_{*}$ in $H^{2 q}\left(\mathrm{BU}_{\mathbb{H}}^{e v} \times \mathbb{P}(\mathbb{H}) ; \mathbb{Z}\right)$ which is multiplication by $(-1)^{q}$, hence

$$
E^{0, q}(n)= \begin{cases}H^{q}\left(\mathrm{BU}_{\mathbb{H}}^{e v} \times \mathbb{P}(\mathbb{H}) ; \mathbb{Z}\right), & q=2 q^{\prime}, n=q^{\prime} \bmod 2, \\ 0, & \text { otherwise. }\end{cases}
$$

It follows from (52) that $\Theta^{0,2 q}(q)$ is an isomorphism. Since $E^{p, 0}(n)=$ $H^{p}(B \mathbb{Z} / 2 ; \mathbb{Z}(n))$, we see that $\Theta^{p, 0}(n)$ is also an isomorphism. By Zeeman's comparison theorem, one concludes that

$$
E_{\infty}^{*, *}(*) \cong F_{\infty}^{*, *}(*)\left[\mathbf{c}_{1}^{\mathbb{H}}, \mathbf{c}_{2}^{\mathbb{H}}, \ldots, \mathbf{c}_{n}^{\mathbb{H}}, \ldots\right] .
$$

A standard argument can be used to show that there is actually a ring isomorphism

$$
\hat{H}_{\mathbb{Z} / 2}^{*}\left(\mathrm{BU}_{\mathbb{H}}^{e v} \times \mathbb{P}(\mathbb{H}) ; \mathbb{Z}(*)\right) \cong \hat{H}_{\mathbb{Z} / 2}^{*}(\mathbb{P}(\mathbb{H}) ; \mathbb{Z}(*))\left[\mathbf{c}_{1}^{\mathbb{H}}, \mathbf{c}_{2}^{\mathbb{H}}, \ldots, \mathbf{c}_{k}^{\mathbb{H}}, \ldots\right] .
$$

By Proposition 1.15] we conclude that

$$
H^{*, *}\left(\mathrm{BU}_{\mathbb{H}}^{e v} \times \mathbb{P}(\mathbb{H}) ; \underline{\mathbb{Z}}\right) \cong H^{*, *}(\mathbb{P}(\mathbb{H}) ; \underline{\mathbb{Z}})\left[\mathbf{c}_{1}^{\mathbb{H}}, \mathbf{c}_{2}^{\mathbb{H}}, \ldots, \mathbf{c}_{k}^{\mathbb{H}}, \ldots\right]
$$

5.2. Projective bundle formula. It is natural to look for a relation between the classes $c_{k}^{\mathbb{H}}(E)$ and the structure of $H^{*, *}(\mathbb{P}(E) \times \mathbb{P}(\mathbb{H}) ; \underline{\mathbb{Z}})$, as a module over $H^{*, *}(X \times \mathbb{P}(\mathbb{H}) ; \underline{\mathbb{Z}})$.

Proposition 5.4. Let $E \stackrel{p}{\rightarrow} X$ be a quaternionic bundle of rank e, and let $\mathbb{P}(E)$ denote its projectivization. If $\xi$ in $K H(\mathbb{P}(E))$ is the universal quotient bundle over $\mathbb{P}(E)$, then $H^{*, *}(\mathbb{P}(E) \times \mathbb{P}(\mathbb{H}) ; \underline{\mathbb{Z}})$ is a free $H^{*, *}(X \times \mathbb{P}(\mathbb{H}) ; \underline{\mathbb{Z}}$-module generated by $c_{k}^{\mathbb{H}}(\xi), k=0, \ldots, e-1$. Moreover, the classes $c_{0}^{\mathbb{H}}(\xi), \ldots, c_{e}^{\mathbb{H}}(\xi)$ satisfy the following relation:

$$
\sum_{k=0}^{e}(-1)^{k} c_{k}^{\mathbb{H}}(\xi) c_{e-k}^{\mathbb{H}}\left(p^{*} E\right)=0
$$

Proof. Let $p_{1}: X \times \mathbb{P}(\mathbb{H}) \rightarrow X$ and $p_{2}: X \times \mathbb{P}(\mathbb{H}) \rightarrow \mathbb{P}(\mathbb{H})$ be the projections. Then $G:=p_{1}^{*}(E) \otimes p_{2}^{*}\left(\mathcal{O}_{\mathbb{P}(\mathbb{H})}(1)\right)$ is a Real bundle, and there is an equivariant homeomorphism

$$
\mathbb{P}(G)=\mathbb{P}\left(p_{1}^{*}(E) \otimes p_{2}^{*}\left(\mathcal{O}_{\mathbb{P}(\mathbb{H})}(1)\right)\right) \cong \mathbb{P}\left(p_{1}^{*}(E)\right) \cong \mathbb{P}(E) \times \mathbb{P}(\mathbb{H}) .
$$

It follows from the projective bundle formula for Real bundles that, if $\mathcal{O}_{G}(1)$ is the dual of the tautological line bundle over $\mathbb{P}(E)$ and $t=c_{1}^{\mathbb{R}}\left(\mathcal{O}_{G}(1)\right)$, then $H^{*, *}(\mathbb{P}(E) \times \mathbb{P}(\mathbb{H}) ; \underline{\mathbb{Z}})$ is a free $H^{*, *}(X \times \mathbb{P}(\mathbb{H}) ; \underline{\mathbb{Z}})$-module generated by $1, t, \ldots, t^{e-1}$. Moreover, the classes $c_{0}^{\mathbb{R}}\left(p^{*} G\right), \ldots, c_{e}^{\mathbb{R}}\left(p^{*} G\right)$ satisfy the relation

$$
\sum_{\substack{0 \leq \delta \leq 1 \\ 0 \leq 2 i+\delta \leq e}}(-1)^{2 i+\delta} t^{2 i+\delta} c_{e-2 i+\delta}^{\mathbb{R}}\left(q^{*} G\right)=0,
$$

where $q: \mathbb{P}(G) \rightarrow X \times \mathbb{P}(\mathbb{H})$ is the bundle projection.

A simple computation shows that the classes $t^{k}$ can be expressed in terms of the classes $c_{k}^{\mathbb{H}}(\xi)$. In fact, denoting the first Chern class of the Real bundle $\mathcal{O}(2)$ by $\mathbf{x}$ as before, we have

$$
t^{2 i+\delta}=c_{2 i+\delta}^{\mathbb{H}}(\xi)+i c_{2 i+\delta-1}^{\mathbb{H}}(\xi) \mathbf{x},
$$

for $0 \leq \delta \leq 1$ and $0 \leq 2 i+\delta \leq e$. 
Hence $H^{*, *}(\mathbb{P}(E) \times \mathbb{P}(\mathbb{H}) ; \underline{\mathbb{Z}})$ is also generated by $1, c_{1}^{\mathbb{H}}(\xi), \ldots, c_{e}^{\mathbb{H}}(\xi)$. It remains to show that (153) holds. Now,

$$
c_{e-(2 i-\delta)}^{\mathbb{H}}\left(p^{*} E\right)=c_{e-(2 i-\delta)}^{\mathbb{R}}\left(q^{*} G\right)-i c_{e-(2 i-\delta)-1}^{\mathbb{R}}\left(q^{*} G\right) \mathbf{x},
$$

for $0 \leq \delta \leq 1$ and $0 \leq 2 i+\delta \leq e$ (where we set $c_{-1}(G):=0$ ). Hence from (54), (55) and the equality $x^{2}=0$, we get

$$
\begin{aligned}
& \sum_{k=0}^{e}(-1)^{k} c_{k}^{\mathbb{H}}(\xi) c_{e-k}^{\mathbb{H}}\left(p^{*} E\right)=\sum_{\substack{0 \leq \delta \leq 1 \\
0 \leq 2 i+\delta \leq e}}(-1)^{2 i+\delta} c_{2 i+\delta}^{\mathbb{H}}(\xi) c_{e-(2 i+\delta)}^{\mathbb{H}}\left(p^{*} E\right) \\
= & \sum_{\substack{0 \leq \delta \leq 1 \\
0 \leq 2 i+\delta \leq e}}(-1)^{2 i+\delta}\left(t^{2 i+\delta}-i t^{2 i+\delta-1} \mathbf{x}\right)\left(c_{e-(2 i+\delta)}^{\mathbb{R}}\left(q^{*} G\right)-i c_{e-(2 i+\delta)-1}^{\mathbb{R}}\left(q^{*} G\right) \mathbf{x}\right) \\
= & \sum_{\substack{0 \leq \delta \leq 1 \\
0 \leq 2 i+\delta \leq e}}^{\mathbb{R}}(-1)^{2 i+\delta-1} i\left(t^{2 i+\delta} c_{e-(2 i+\delta)-1}^{\mathbb{R}}\left(q^{*} G\right)-t^{2 i+\delta-1} c_{e-(2 i+\delta)}^{\mathbb{R}}\left(q^{*} G\right) \mathbf{x}\right)=0 .
\end{aligned}
$$

5.3. The quaternionic total Chern class map. In this section we will compute the cohomology classes determined by the map $c^{\mathbb{H}}$ and the splitting (40) of Theorem 3.7 .

Proposition 5.5. Let $\xi^{n} \in K H\left(\mathbb{P}\left(\mathbb{H}^{n}\right)\right)$ be the universal quotient bundle over $\mathbb{P}(\mathbb{H})$ and

$$
\psi_{n}: z_{0}\left(\mathbb{P}\left(\mathbb{H}^{n}\right)\right) \rightarrow \prod_{i=1}^{n} F\left(\mathbb{P}(\mathbb{H})_{+}, K(\mathbb{Z}(2 i-1), 4 i-2)\right)
$$

be the equivariant homotopy equivalence of Corollary 2.4. If $j_{n}: \mathbb{P}\left(\mathbb{H}^{n}\right) \hookrightarrow$ $z_{0}\left(\mathbb{P}\left(\mathbb{H}^{n}\right)\right)$ denotes the natural inclusion, then the composition

$$
\phi_{n}:=\psi_{n} \circ j_{n}: \mathbb{P}\left(\mathbb{H}^{n}\right) \rightarrow \prod_{i=1}^{n} F\left(\mathbb{P}(\mathbb{H})_{+}, K(\mathbb{Z}(2 i-1), 4 i-2)\right)
$$

classifies $c_{1}^{\mathbb{H}}\left(\xi^{n}\right), c_{3}^{\mathbb{H}}\left(\xi^{n}\right), \ldots, c_{2 n-1}^{\mathbb{H}}\left(\xi^{n}\right)$.

Proof. For $n=1$, we need to identify the element $\alpha$ of $H^{2,1}(\mathbb{P}(\mathbb{H}) \times \mathbb{P}(\mathbb{H}) ; \underline{\mathbb{Z}})$ classified by the composition $\mathbb{P}(\mathbb{H}) \stackrel{j}{\rightarrow} z_{0}(\mathbb{P}(\mathbb{H})) \stackrel{\psi}{\rightarrow} F\left(\mathbb{P}(\mathbb{H})_{+}, K(\mathbb{Z}(1), 2)\right.$. Since $\psi$ realizes Poincaré duality, $\alpha$ is the Poincaré dual of the diagonal $\Delta \subset \mathbb{P}(\mathbb{H}) \times \mathbb{P}(\mathbb{H})$. From the projective bundle formula (53) it is easy to see that

$$
\alpha=\mathcal{P}(\Delta)=c_{1}\left(p r_{1}^{*} \mathcal{O}(1) \otimes p r_{2}^{*} \mathcal{O}(1)\right)=c_{1}^{\mathbb{H}}\left(\xi^{1}\right) .
$$

Assume that the proposition holds for $k<n$, and note that Proposition 2.2 (c) implies that $\phi_{n}$ restricts to $\phi_{k}$ on $\mathbb{P}\left(\mathbb{H}^{k}\right)$, for $k<n$. The projective bundle formula shows that $c_{2 i-1}^{\mathbb{H}}\left(\xi^{n}\right)$ is the only class whose restriction to $\mathbb{P}\left(\mathbb{H}^{k}\right)$ is $c_{2 i-1}^{\mathbb{H}}\left(\xi^{k}\right)$, for all $i=1, \ldots, k$. Hence it suffices to show that $p_{n} \circ \phi_{n}$ classifies $c_{n}^{\mathbb{H}}\left(\xi^{n}\right)$, where $p_{n}$ denotes the projection onto the last factor in (57).

Observe that $p_{n} \circ \phi_{n}$ is given by the composition

$$
\begin{aligned}
& \mathbb{P}\left(\mathbb{H}^{n}\right)_{+} \stackrel{\pi}{\rightarrow} \mathbb{P}\left(\mathbb{H}^{n}\right) / \mathbb{P}\left(\mathbb{H}^{n-1}\right) \stackrel{j}{\rightarrow} z_{0}\left(\mathbb{P}\left(\mathbb{H}^{n}\right) / \mathbb{P}\left(\mathbb{H}^{n-1}\right)\right)_{\mathrm{o}} \\
& \stackrel{\theta}{\rightarrow} F\left(\mathbb{P}(\mathbb{H})_{+}, K(\mathbb{Z}(2 n-1), 4 n-2)\right),
\end{aligned}
$$


where $\pi$ is the projection, $j$ is the natural inclusion and $\theta$ is the equivalence given in (17). The adjoint map $(\theta \circ j)^{\vee}: \frac{\mathbb{P}\left(\mathbb{H}^{n}\right) \times \mathbb{P}(\mathbb{H})}{\mathbb{P}\left(\mathbb{H}^{n-1}\right) \times \mathbb{P}(\mathbb{H})} \rightarrow K(\mathbb{Z}(2 n-1), 4 n-2)$ can be described as the composition

$$
\begin{gathered}
\mathrm{T}\left(\mathcal{O}(1) \otimes \mathbb{H}^{n-1}\right) \wedge \mathbb{P}(\mathbb{H})_{+} \\
\stackrel{\Delta \wedge i d}{\longrightarrow}\left(\mathrm{T}\left(\mathcal{O}(1) \otimes \mathbb{H}^{n-1}\right) \wedge \mathbb{P}(\mathbb{H})_{+}\right) \wedge \mathbb{P}(\mathbb{H})_{+}=\mathrm{T}\left(\mathcal{O}(1) \otimes \mathbb{H}^{n-1}\right) \wedge(\mathbb{P}(\mathbb{H}) \wedge \mathbb{P}(\mathbb{H}))_{+} \\
\stackrel{f_{\mu} \wedge c_{1}^{\mathbb{H}}}{\longrightarrow} Z_{0}\left(S^{4(n-1), 2(n-1)}\right)_{\mathrm{o}} \wedge Z_{0}\left(S^{2,1}\right)_{\mathrm{o}} \rightarrow Z_{0}\left(S^{4 n-2,2 n-1}\right)_{\mathrm{o}}=K(\mathbb{Z}(2 n-1), 4 n-2),
\end{gathered}
$$

where $f_{\mu}$ classifies the Thom class of $\mathcal{O}(1) \otimes \mathbb{H}^{n-1}$. One can easily check that the pull-back of $\left[f_{\mu}\right]$ under the projection $\pi: \mathbb{P}\left(\mathbb{H}^{n}\right) \rightarrow \mathrm{T}\left(\mathcal{O}(1) \otimes \mathbb{H}^{n-1}\right)$ coincides with $c_{2 n-2}^{\mathbb{H}}\left(\xi^{n}\right)$. Therefore, $\left[p_{n} \circ \phi_{n}\right]=c_{2 n-2}^{\mathbb{H}}\left(\xi^{n}\right) c_{1}^{\mathbb{H}}\left(\xi^{n}\right)=c_{2 n-1}^{\mathbb{H}}\left(\xi^{n}\right)$, and this completes the proof of the proposition.

We can now compute the classes determined by the total quaternionic Chern class map $c^{\mathbb{H}}$.

Theorem 5.6. The equivariant cohomology classes determined by total quaternionic Chern class map $c^{\mathbb{H}}:(\mathbb{Z} \times B U)_{\mathbb{H}} \rightarrow \mathcal{Z}_{\mathbb{H}}$ and the splitting (40) of Theorem 3.7] are

$$
\begin{aligned}
\mathbf{1}+\mathbf{c}_{2}^{\mathbb{H}}+\mathbf{c}_{4}^{\mathbb{H}}+\cdots+\mathbf{c}_{2 n}^{\mathbb{H}}+\cdots & \text { on } B U_{\mathbb{H}}^{e v}, \\
\mathbf{c}_{1}^{\mathbb{H}}+\mathbf{c}_{3}^{\mathbb{H}}+\cdots+\mathbf{c}_{2 n+1}^{\mathbb{H}}+\cdots & \text { on } B U_{\mathbb{H}}^{\text {odd }} .
\end{aligned}
$$

Proof. Recall that there are natural equivalences $\mathrm{BU}_{\mathbb{H}}^{j} \cong \mathrm{BU}_{\mathbb{H}}^{j+2}$ and $z_{\mathbb{H}}^{j} \cong z_{\mathbb{H}}^{j+2}$ and, moreover, the map $c^{\mathbb{H}}:(\mathbb{Z} \times B U)_{\mathbb{H}} \rightarrow \mathcal{Z}_{\mathbb{H}}$ is compatible with these equivalences. Thus $c^{\mathbb{H}}$ induces maps

$$
c_{e v}^{\mathbb{H}}: \mathrm{BU}_{\mathbb{H}}^{e v} \rightarrow z_{\mathbb{H}}^{\mathrm{ev}} \quad \text { and } \quad c_{o d d}^{\mathbb{H}}: \mathrm{BU}_{\mathbb{H}}^{\text {odd }} \rightarrow z_{\mathbb{H}}^{\text {odd }}
$$

and it suffices to compute the equivariant cohomology classes they classify.

The map $c_{\text {odd }}^{\mathbb{H}}: \mathrm{BU}_{\mathbb{H}}^{\text {odd }} \rightarrow Z_{\mathbb{H}}^{\text {odd }}$ classifies an element $D_{1}+D_{3}+\cdots+D_{2 n-1}+\cdots$ with

$$
D_{2 i-1} \in H^{4 i-2,2 i-1}\left(\mathrm{BU}_{\mathbb{H}}^{\text {odd }} \times \mathbb{P}(\mathbb{H}) ; \underline{\mathbb{Z}}\right), i \geq 1 .
$$

Note that by construction, we have $c^{\mathbb{H}}\left(\mathrm{Gr}^{2 q-3}\left(\mathbb{H}^{k^{*}} \oplus \mathbb{H}^{k}\right)\right) \subset \mathcal{Z}_{\mathbb{H}}^{2 q-3}\left(\mathbb{H}^{k^{*}} \oplus \mathbb{H}^{k}\right)$, hence

$$
\left(D_{2 q-1}\right)_{\mid \operatorname{Gr}^{2 q-3}\left(\mathbb{H}^{k *} \oplus \mathbb{H}^{k}\right)}=0, \quad k \geq 0 .
$$

It follows from Theorem 5.3 that there are constants $\lambda_{1}, \lambda_{2}$ such that

$$
D_{2 q-1}=\lambda_{1} \mathbf{c}_{2 q-1}^{\mathbb{H}}+\lambda_{2} \mathbf{x} \cdot \mathbf{c}_{2 q}^{\mathbb{H}},
$$

where $\mathbf{x} \in H^{2,1}(\mathbb{P}(\mathbb{H}) ; \underline{\mathbb{Z}})$ is the fundamental class of $\mathbb{P}(\mathbb{H})$. To compute $\lambda_{1}, \lambda_{2}$ we observe that, by Proposition 5.5 the restriction of $D_{2 q-1}$ to $\mathbb{P}\left(\mathbb{H}^{q}\right)$ is $c_{2 q-1}^{\mathbb{H}}\left(\xi^{q}\right)$, where $\xi^{q}$ is the universal quotient bundle over $\mathbb{P}\left(\mathbb{H}^{q}\right)$. Since the inclusion

$$
\mathbb{P}\left(\mathbb{H}^{q}\right) \subset \mathrm{Gr}^{2 q-1}\left(\mathbb{H}^{q *}\right) \mathrm{Gr}^{2 q-1}\left(\mathbb{H}^{q *}\right) \subset \mathrm{Gr}^{2 q-1}\left(\mathbb{H}^{q *} \oplus \mathbb{H}^{q}\right) \subset \mathrm{BU}_{\mathbb{H}}^{2 q-1}
$$

classifies $\xi^{q}$, it follows that $D_{2 q-1 \mid \mathbb{P}\left(\mathbb{H}^{q}\right)}=c_{2 q-1}^{\mathbb{H}}\left(\xi^{q}\right)$. Thus $\lambda_{1}=1, \lambda_{2}=0$ and $D_{2 q-1}=\mathbf{c}_{2 q-1}^{\mathbb{H}}$. 
Now, consider the map $c_{e v}^{\mathbb{H}}: \mathrm{BU}_{\mathbb{H}}^{e v} \rightarrow z_{\mathbb{H}}^{\text {ev }}$. It classifies an element $1+D_{2}+D_{4}+$ $\cdots+D_{2 n}+\cdots$ with $D_{2 i} \in H^{4 i, 2 i}\left(\mathrm{BU}_{\mathbb{H}}^{e v} \times \mathbb{P}(\mathbb{H}) ; \underline{\mathbb{Z}}\right), i \geq 1$. Once again, we observe that $c^{\mathbb{H}}\left(\mathrm{Gr}^{2 q-2}\left(\mathbb{H}^{k^{*}} \oplus \mathbb{H}^{k}\right)\right) \subset \mathcal{Z}_{\mathbb{H}}^{2 q-2}\left(\mathbb{H}^{k^{*}} \oplus \mathbb{H}^{k}\right)$, implying

$$
\left(D_{2 q-2}\right)_{\mid \mathrm{Gr}^{2 q-2}\left(\mathbb{H}^{k *} \oplus \mathbb{H}^{k}\right)}=0, \quad k \geq 0 .
$$

As before, we conclude that there are constants $\lambda_{1}, \lambda_{2}$ such that $D_{2 q}=\lambda_{1} \mathbf{c}_{2 q}^{\mathbb{H I}}+$ $\lambda_{2} \mathbf{x} \cdot \mathbf{c}_{2 q-1}^{\mathbb{H}}$. To compute $\lambda_{1}, \lambda_{2}$ it suffices to compute the image of $D_{2 q}$ under the forgetful map to singular cohomology. In [LM88] it is shown that, non-equivariantly, $z_{1}\left(\mathbb{P}\left(\mathbb{H}^{n}\right)\right)_{1} \cong K(\mathbb{Z}, 2) \times \cdots \times K(\mathbb{Z}, 4 n-2)$, and that under this equivalence $c_{\mathbb{H}}$ classifies the total Chern class. One can show that the decomposition (33) is compatible with this non-equivariant splitting. It follows that $\rho\left(D_{2 k}\right)=\mathbf{c}_{k}+\mathbf{c}_{k-1} \beta$, hence $\lambda_{1}=1$ and $\lambda_{2}=0$.

5.4. The group struture on $\mathfrak{Z}_{\mathbb{H}}^{0}(X)$. In this section we compute the group structure induced by the algebraic join of cycles on $z_{\mathbb{H}}$. Recall from Proposition 3.6 that the algebraic join \# induces a pairing

$$
\#: z_{\mathbb{H}} \times z_{\mathbb{H}} \rightarrow z_{\mathbb{H}}
$$

satisfying

$$
\#: z_{\mathbb{H}}^{j} \times z_{\mathbb{H}}^{j} \rightarrow z_{\mathbb{H}}^{j+j^{\prime}}
$$

For a Real space $X$, one has an identification

$$
\mathfrak{Z}_{\mathbb{H}}^{0}(X)=\left[X, \mathcal{Z}_{\mathbb{H}}\right]_{\mathbb{Z} / 2}=\bigoplus_{j \in \mathbb{Z}}\left[X, Z_{\mathbb{H}}^{j}\right]_{\mathbb{Z} / 2} \subseteq \bigoplus_{j \in \mathbb{Z}} \prod_{r \geq 1} H^{4 r-2 \epsilon(j), 2 r-\epsilon(j)}(X \times \mathbb{P}(\mathbb{H}), \underline{\mathbb{Z}}),
$$

where $\epsilon(j)$ is 0 if $j$ is even and 1 if $J$ is odd. Given the splitting above one might conjecture that the group structure induced by \# is induced by the cup-product on $H^{*, *}(X \times \mathbb{P}(\mathbb{H}) ; \underline{\mathbb{Z}})$; however this is not the case as we will show. From (61) it follows that the group structure on $\mathfrak{Z}_{\mathbb{H}}^{0}(X)$ is completely determined by the cohomology class represented by the map \# under the inclusions

$$
\left[Z_{\mathbb{H}}^{j} \times Z_{\mathbb{H}}^{j^{\prime}}, Z_{\mathbb{H}}^{j+j^{\prime}}\right]_{\mathbb{Z} / 2} \subseteq \prod_{r \geq 1} H^{4 r-2 \epsilon\left(j+j^{\prime}\right), 2 r-\epsilon\left(j+j^{\prime}\right)}\left(Z_{\mathbb{H}}^{j} \times Z_{\mathbb{H}}^{j^{\prime}} \times \mathbb{P}(\mathbb{H}), \underline{\mathbb{Z}}\right) .
$$

Also recall that $\mathrm{BU}_{\mathbb{H}}^{j}$ maps to $\mathcal{Z}_{\mathbb{H}}^{j}$ by $c_{\mathbb{H}}$ and that the following diagram commutes:

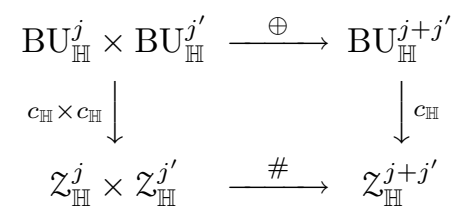

We claim that the maps $c_{\mathbb{H}}$ above induce injective maps

$$
H^{*, *}\left(\mathcal{Z}_{\mathbb{H}}^{j} \times \mathbb{P}(\mathbb{H}) ; \underline{\mathbb{Z}}\right) \rightarrow H^{*, *}\left(\mathcal{Z}_{0}\left(\mathrm{BU}_{\mathbb{H}}^{j}\right)_{1} \times \mathbb{P}(\mathbb{H}) ; \underline{\mathbb{Z}}\right)
$$

and hence the pairing on $\mathfrak{Z}_{\mathbb{H}}^{0}(-)$ is completely determined by the formula for the quaternionic Chern class of a Whitney sum.

Let us start with the case $j=1$. Let $\iota: \mathbb{P}\left(\mathbb{H}^{\infty}\right) \rightarrow \mathrm{BU}_{\mathbb{H}}^{1}$ be the map induced by the inclusions $\iota_{n}: \mathbb{P}\left(\mathbb{H}^{n}\right) \rightarrow \mathrm{Gr}^{1}\left(\mathbb{H}^{n *} \oplus \mathbb{H}^{n}\right)$ that send $L$ to $L \oplus \mathbb{H}^{n}$. Composing with $c_{\mathbb{H}}$ gives a map from $\mathbb{P}\left(\mathbb{H}^{\infty}\right)$ to $z_{\mathbb{H}}^{1}$. The linear extension $z_{0}\left(\mathbb{P}\left(\mathbb{H}^{n}\right)\right) \rightarrow z_{\mathbb{H}}^{1}$ of this composition is, by the quaternionic suspension theorem, an equivariant homotopy equivalence when restricted to the component of 1 . This map factors as 
$z_{0}\left(\mathbb{P}\left(\mathbb{H}^{n}\right)\right)_{1} \stackrel{\widetilde{\iota}}{\rightarrow} z_{0}\left(\mathrm{BU}_{\mathbb{H}}^{1}\right)_{1} \stackrel{\widetilde{c_{\mathbb{H}}}}{\longrightarrow} z_{\mathbb{H}}^{1}$, where $\widetilde{\iota}$ and $\widetilde{c_{\mathbb{H}}}$ denote the linear extension of $\iota$ and $c_{\mathbb{H}}$, respectively. It follows that, for any cohomology theory $\mathfrak{H}^{*}$, the map $\mathfrak{H}\left(\widetilde{c_{\mathbb{H}}}\right): \mathfrak{H}^{*}\left(Z_{\mathbb{H}}^{1}\right) \rightarrow \mathfrak{H}^{*}\left(Z_{0}\left(\mathrm{BU}_{\mathbb{H}}^{1}\right)_{1}\right)$ is injective. For $1 \neq j$ odd it suffices to observe that $\widetilde{c_{\mathbb{H}}}$ is compatible with the canonical equivariant homotopy equivalence $z_{\mathbb{H}}^{j} \cong z_{\mathbb{H}}^{j+2}$, given by inclusion of hyperplanes.

Suppose now that $j$ is even. We need to show that $\widetilde{c_{\mathbb{H}}}$ induces an injective map

$$
\left.\left(\operatorname{id} \times \widetilde{c_{\mathbb{H}}}\right)^{*}: H^{*, *}\left(\mathcal{Z}_{\mathbb{H}}^{j} \times \mathbb{P}(\mathbb{H}) ; \underline{\mathbb{Z}}\right) \rightarrow H^{*, *}\left(\mathcal{Z}_{0}\left(\mathrm{BU}_{\mathbb{H}}^{j}\right)_{1}\right) \times \mathbb{P}(\mathbb{H}) ; \underline{\mathbb{Z}}\right) .
$$

The same argument shows that we may replace $\mathrm{BU}_{\mathbb{H}}^{j}$ by $\mathrm{BU}_{\mathbb{H}}^{e v}$. The homotopy equivalences $\Psi$ and $\Phi$ from Remark 5.2 are compatible with $c_{\mathbb{H}}$, hence the following diagram commutes:

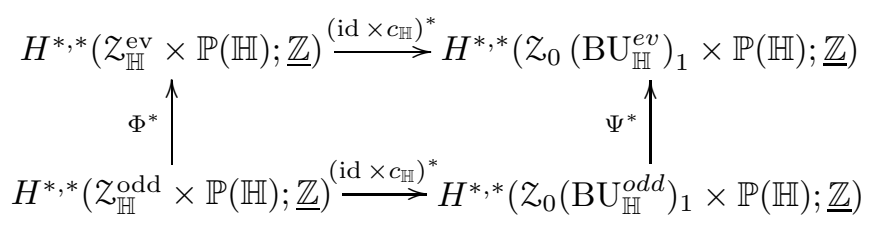

It follows that the map on top is injective.

Proposition 5.7. Let $X$ be a Real space, and let $a \cdot b$ denote the product of elements a,b in $\mathfrak{Z}_{\mathbb{H}}^{0}(X)$. Consider $\mathfrak{Z}_{\mathbb{H}}^{0}(X)$ included in

$$
\bigoplus_{j \in \mathbb{Z}} \prod_{r, s \geq 1} H^{r, s}(X \times \mathbb{P}(\mathbb{H}), \underline{\mathbb{Z}}),
$$

as in (61). Then, under this inclusion we have,

$$
a \cdot b=a \cup b+p r^{*}(a / \mathbf{z}) \cup p r^{*}(b / \mathbf{z}),
$$

where $\mathbf{z} \in H_{2,1}(\mathbb{P}(\mathbb{H}) ; \underline{\mathbb{Z}})$ is the fundamental class of $\mathbb{P}(\mathbb{H}),-/ \mathbf{z}$ denotes slant product with $z$ and pr is the projection onto the first factor in the product $X \times \mathbb{P}(\mathbb{H})$.

Proof. By the preceding remarks it suffices to show that the following formula for the quaternionic Chern class of a Whitney sum holds:

$$
c_{\mathbb{H}}(E \oplus F)=c_{\mathbb{H}}(E) \cup c_{\mathbb{H}}(F)+p r^{*}\left(c_{\mathbb{H}}(E) / \mathbf{z}\right) \cup p r^{*}\left(c_{\mathbb{H}}(F) / \mathbf{z}\right) .
$$

Recall that $c_{\mathbb{H}}(E)$ is defined as a combination of Chern classes of the Real bundle $E \otimes \mathcal{O}(1)$. To simplify notation we will use $\bar{E}$ and $\bar{F}$ to denote $E \otimes \mathcal{O}(1)$ and $F \otimes \mathcal{O}(1)$, respectively. Let $e, f$ be the dimensions of $E$ and $F$, respectively. We have

$$
\begin{aligned}
& c_{\mathbb{H}}(E) \cup c_{\mathbb{H}}(F)=\sum_{i=0}^{\left\lfloor\frac{e}{2}\right\rfloor}\left[c_{e-2 i}^{\mathbb{R}}(\bar{E})-i c_{e-2 i-1}^{\mathbb{R}}(\bar{E}) \mathbf{x}\right] \cup \sum_{j=0}^{\left\lfloor\frac{f}{2}\right\rfloor}\left[c_{f-2 j}^{\mathbb{R}}(\bar{F})-j c_{f-2 j-1}^{\mathbb{R}}(\bar{F}) \mathbf{x}\right] \\
&=\sum_{r=0}^{\left\lfloor\frac{e+f}{2}\right\rfloor} \sum_{s=0}^{r} c_{e-2 s}^{\mathbb{R}}(\bar{E}) \cup c_{f-2(r-s)}^{\mathbb{R}}(\bar{F}) \\
&-\left[s c_{e-2 s-1}^{\mathbb{R}}(\bar{E}) \cup c_{f-2(r-s)}^{\mathbb{R}}(\bar{F})+(r-s) c_{e-2 s}^{\mathbb{R}}(\bar{E}) \cup c_{f-2(r-s)-1}^{\mathbb{R}}(\bar{F})\right] \mathbf{x},
\end{aligned}
$$


where we set $c_{k}^{\mathbb{R}}(-)$ equal to 1 if $k$ is zero and $c_{k}^{\mathbb{R}}(-)$ equal to zero if $k<0$. By Lemma 5.8 below, we have

$$
\begin{aligned}
p^{*}\left(c_{\mathbb{H}}(E) / \mathbf{z}\right) \cup p r^{*}\left(c_{\mathbb{H}}(F) / \mathbf{z}\right) \\
=\sum_{i=0}^{\left\lfloor\frac{e}{2}\right\rfloor} c_{e-2 i-1}^{\mathbb{H}}(E) \cup \sum_{j=0}^{\left\lfloor\frac{f}{2}\right\rfloor} c_{f-2 j-1}^{\mathbb{H}}(F) \\
=\sum_{r=0}^{\left\lfloor\frac{e+f}{2}\right\rfloor} \sum_{s=0}^{r} c_{e-2 s-1}^{\mathbb{R}}(\bar{E}) \cup c_{f-2(r-s)-1}^{\mathbb{R}}(\bar{F}) \\
-\left[s c_{e-2 s-2}^{\mathbb{R}}(\bar{E}) \cup c_{f-2(r-s)-1}^{\mathbb{R}}(\bar{F})+(r-s) c_{e-2 s-1}^{\mathbb{R}}(\bar{E}) \cup c_{f-2(r-s)-2}^{\mathbb{R}}(\bar{F})\right] \mathbf{x} .
\end{aligned}
$$

Thus, we get

$$
\begin{aligned}
& c_{\mathbb{H}}(E) \cup c_{\mathbb{H}}(F)+p r^{*}\left(c_{\mathbb{H}}(E) / \mathbf{z}\right) \cup p r^{*}\left(c_{\mathbb{H}}(F) / \mathbf{z}\right) \\
&= \sum_{r=0}^{\left\lfloor\frac{e+f}{2}\right\rfloor} \sum_{s=0}^{2 r} c_{e-s}^{\mathbb{R}}(\bar{E}) \cup c_{f-2 r+s}^{\mathbb{R}}(\bar{F}) \\
& \quad-\left[r c_{e-2 s-1}^{\mathbb{R}}(\bar{E}) \cup c_{f-2 r+s}^{\mathbb{R}}(\bar{F})+r c_{e-2 s}^{\mathbb{R}}(\bar{E}) \cup c_{f-2 r+s-1}^{\mathbb{R}}(\bar{F})\right] \mathbf{x} \\
&= \\
& \sum_{r=0}^{\left\lfloor\frac{e+f}{2}\right\rfloor} c_{e+f-2 r}^{\mathbb{R}}(\bar{E} \oplus \bar{F})-r c_{e+f-2 r-1}^{\mathbb{R}}(\bar{E} \oplus \bar{F}) \mathbf{x}=c_{\mathbb{H}}(E \oplus F) .
\end{aligned}
$$

The proposition follows.

Lemma 5.8. Let $E \rightarrow X$ be a quaternionic bundle of dimension $e$, and let $\mathbf{z} \in$ $H_{2,1}(\mathbb{P}(\mathbb{H}) ; \mathbb{Z})$ be the fundamental homology class. Then we have

$$
\operatorname{pr}^{*}\left(c_{e-2 k}^{\mathbb{H}}(E) / \mathbf{z}\right)=c_{e-2 k-1}^{\mathbb{H}}(E), \quad k=0, \ldots,\left\lfloor\frac{e-2}{2}\right\rfloor,
$$

where $-/ \mathbf{z}$ denotes slant product with $\mathbf{z}$ and $p r$ is projection to the first factor of $X \times \mathbb{P}(\mathbb{H})$.

Proof. We will prove the case where $e$ is even. The other case is proven similarly. Recall from Theorem 5.3 that the equivariant cohomology of $\mathrm{BU}_{\mathbb{H}}^{e v} \times \mathbb{P}(\mathbb{H})$ is generated over $H^{*, *}(\mathbb{P}(\mathbb{H}) ; \underline{\mathbb{Z}})$ by the classes $\mathbf{c}_{k}^{\mathbb{H}}, k \geq 1$. The result will follow once we prove the identity

$$
\operatorname{pr}^{*}\left(\mathbf{c}_{2 k}^{\mathbb{H}} / \mathbf{z}\right)=\mathbf{c}_{2 k-1}^{\mathbb{H}},
$$

where $p r: \mathrm{BU}_{\mathbb{H}}^{e v} \times \mathbb{P}(\mathbb{H})$ is the projection onto the first factor. Observe that, by construction, the restriction of $\mathbf{c}_{2 k}^{\mathbb{H}}$ to $\mathrm{Gr}^{2 k-2}\left(\mathbb{H}^{r *} \oplus \mathbb{H}^{s}\right)$ is zero. Hence, by Theorem 5.3, $\operatorname{pr}^{*}\left(\mathbf{c}_{2 k}^{\mathbb{H}} / \mathbf{z}\right)$ must be a multiple of $\mathbf{c}_{2 k-1}^{\mathbb{H}}$. Since

$$
\rho\left(p r^{*}\left(\mathbf{c}_{2 k}^{\mathbb{H}} / \mathbf{z}\right)\right)=\rho\left(\mathbf{c}_{2 k-1}^{\mathbb{H}}\right)=\mathbf{c}_{2 k-1} \times 1,
$$

it follows that $p r^{*}\left(\mathbf{c}_{2 k}^{\mathbb{H}} / \mathbf{z}\right)=\mathbf{c}_{2 k-1}^{\mathbb{H}}$. 


\section{Appendix A. Equivariant $\mathcal{J}_{*}$-FUnCtors}

In this appendix we recall the notion of equivariant $\mathcal{J}_{*}$-functors and the "recognition principle" for equivariant infinite loop spaces developed by Costenoble and Waner in CW91. Let $G$ be a finite group and let $\mathcal{T}_{G}$ denote the full subcategory of the category of based spaces whose objects are all based $G$-spaces. The category $\mathcal{T}_{G}$ is enriched over the category $G \mathcal{T}$ of $G$-spaces and $G$-maps in the sense that the morphism space $\operatorname{Mor}_{\mathcal{T}_{G}}(X, Y):=\mathcal{M} \mathfrak{a} \mathfrak{p}(X, Y)$ becomes a $G$-space under conjugation, in the usual fashion.

Let $G J_{*}$ denote the category whose objects are finite dimensional real representations of $G$, or $\mathbb{R}[G]$-modules, and whose morphisms are (non-equivariant) linear embeddings of real vector spaces. Once again, this category is enriched over the category of $\mathbb{R}[G]$-modules, since $\operatorname{Mor}_{G \mathcal{J}_{*}}(V, W)$ becomes an $\mathbb{R}[G]$-module under the conjugation operation, for all $\mathbb{R}[G]$-modules $V$ and $W$.

Remark A.1. In the non-equivariant context May77, Def. 1.8] May defines the category $\mathcal{J}_{*}$ by requiring the objects to be finite dimensional real vector spaces $(V,\langle-,-\rangle)$ along with an inner product and the morphisms to be isometric embeddings. In the same fashion, we can restrict our category $G J_{*}$ to have orthogonal $\mathbb{R}[G]$-modules as objects and isometric embeddings as morphisms. In the present context we do not need this restriction.

Definition A.2. A $G \mathcal{J}_{*}$-functor is a continuous covariant functor $T: G \mathcal{J}_{*} \rightarrow \mathcal{T}_{G}$ together with a (coherently) commutative, associative and continuous natural transformation

$$
\omega: T \times T \rightarrow T \circ \oplus
$$

satisfying the following conditions. For $\mathbb{R}[G]$-modules $V$ and $W$ one has:

(i) If $x \in T V$ and if $\mathbf{1} \in T\{0\}$ is the base point, then $\omega(x, \mathbf{1})=x \in T V \equiv$ $T(V \oplus\{0\})$. (The identification $T V \equiv T(V \oplus\{0\})$ is determined by the natural inclusion $V \hookrightarrow V \oplus\{0\}$.)

(ii) If $V=V^{\prime} \oplus V^{\prime \prime}$, then the map $T V^{\prime} \rightarrow T V$ given by $x \mapsto \omega(x, 1)$ is a homeomorphism onto a closed subset, where $\mathbf{1} \in T V^{\prime \prime}$ denotes the base point.

(iii) (Enriched functor) The map

$$
T: \operatorname{Mor}_{G \mathcal{J}_{*}}(V, W) \rightarrow \mathcal{M} \mathfrak{a p}(T V, T W)
$$

is a $G$-map.

(iv) The map $\omega: T V \times T W \rightarrow T(V \oplus W)$ is a $G$-map.

The primary use of $G \mathcal{J}_{*}$-functors is to provide a simple and convenient way to build $G$-spaces with an action of the equivariant linear isometries operad, as we now explain. First, let $\mathcal{U}$ be a complete $G$-universe; in other words, $\mathcal{U}$ is an orthogonal $\mathbb{R}[G]$-module containing infinitely countably many copies of each irreducible $\mathbb{R}[G]$ module.

Definition A.3. Given a complete $G$-universe $\mathcal{U}$, the equivariant linear isometries operad $\mathcal{L}_{*}(\mathcal{U})$ is the collection $\left\{\mathcal{L}_{n}:=\operatorname{Isom}\left(\mathcal{U}^{\oplus n}, \mathcal{U}\right) \mid n=0,1,2, \ldots\right\}$, where $\operatorname{Isom}\left(\mathcal{U}^{\oplus n}, \mathfrak{U}\right)$ consists of all (non-equivariant) linear isometric embeddings of $\mathcal{U} \oplus$ $\cdots \oplus \mathcal{U}(n$ copies $)$ into $\mathcal{U}$. The spaces $\mathcal{L}_{n}$ are $\left(G \times \mathfrak{S}_{n}\right)$-spaces, for the right action of the symmetric group $\mathfrak{S}_{n}$ by permuting coordinates and the left action of $G$ by 
conjugation via the diagonal action on $\mathcal{U}^{\oplus n}$. The collection $\mathcal{L}_{*}(\mathcal{U})$ comes with $G$-equivariant maps

$$
\Theta_{n}: \mathcal{L}_{n} \times \mathcal{L}_{k_{1}} \times \cdots \times \mathcal{L}_{k_{n}} \longmapsto \mathcal{L}_{k_{1}+\cdots+k_{n}},
$$

satisfying well-known compatibility conditions. See CW91 Def. 2.1] for the general definition of a $G$-operad and [CW91. Ex. 2.2] for the definition of the $G$-linear isometries operad.

Remark A.4. When $\mathcal{U}$ is a complete $G$-universe, $\mathcal{L}_{*}(\mathcal{U})$ is an example of a $C W E_{\infty}$ $G$-operad; cf. [CW91, Def. 2.3].

One says that a $G$-space $X$ has an action of the operad $\mathcal{L}_{*}(\mathcal{U})$ if one has $\left(G \times \mathfrak{S}_{n}\right)$ equivariant maps

$$
\theta_{n}: \mathcal{L}_{n} \times X^{\times n} \rightarrow X,
$$

where $\mathfrak{S}_{n}$ permutes coordinates in $X^{\times n}$ and acts trivially on $X$. The collection of maps $\theta_{n}$ must satisfy certain coherence conditions; cf. [CW91, Def. 2.4] or [May72.

Now, let $T: G \mathcal{J}_{*} \rightarrow \mathcal{T}_{G}$ be a $G \mathcal{J}_{*}$-functor, and define

$$
T(\mathcal{U}):=\lim _{\overrightarrow{V \subset \mathcal{U}}} T V,
$$

where the colimit is taken over the directed system consisting of all finite dimensional linear subspaces of $\mathcal{U}$ and their natural inclusions.

The following result is an equivariant analogue of [May77, Def. 1.6].

Proposition A.5. Let $T: G \mathcal{J}_{*} \rightarrow \mathcal{T}_{G}$ be a $G J_{*}$-functor. Then $T(\mathcal{U})$ is a $G$-space with a natural action of the equivariant linear isometries operad $\mathcal{L}_{*}(\mathcal{U})$.

Proof. First observe that the family $\mathcal{F}$ of finite dimensional $\mathbb{R}[G]$-submodules of $\mathcal{U}$ is cofinal in the family of all finite dimensional linear subspaces of $\mathcal{U}$. It follows from Definition A.2(iii) that given an inclusion $j: V \hookrightarrow W$ with $V, W \in \mathcal{F}$ the map $j_{W}^{V}:=T(j): T V \rightarrow T W$ is $G$-equivariant, and this immediately implies that $T(\mathcal{U})$ is a $G$-space.

Given $W \in \mathcal{F}$, denote by $j_{W}: T W \rightarrow T(\mathcal{U})$ the natural map from $T W$ to the colimit $T(\mathcal{U})$. In particular, if $V \in \mathcal{F}$ satisfies $V \subset W$ one has $j_{V}=j_{W} \circ j_{W}^{V}$. Following [May77] we define maps

$$
\theta_{n}: \mathcal{L}_{n} \times T(\mathcal{U})^{\times n} \longrightarrow T(\mathcal{U})
$$

as follows. Given $f: \mathcal{U}^{\oplus n} \rightarrow \mathcal{U}$ in $\mathcal{L}_{n}$ and $\left(\sigma_{1}, \ldots, \sigma_{n}\right) \in T(\mathcal{U})^{\times n}$, one can find $V_{1}, \ldots, V_{n} \in \mathcal{F}$ and elements $\mathbf{a}_{i} \in T V_{i}$ such that $j_{V_{i}}\left(\mathbf{a}_{i}\right)=\sigma_{i}$, for all $i=1, \ldots, n$. We can find $W \in \mathcal{F}$ such that $f\left(V_{1} \oplus \cdots \oplus V_{n}\right) \subset W$, hence $f$ induces an embedding $\widehat{f}: V_{1} \oplus \cdots \oplus V_{n} \rightarrow W$. Define

$$
\theta_{n}\left(f ; \sigma_{1}, \ldots, \sigma_{n}\right):=j_{W} \circ T(\widehat{f})\left(\omega\left(\mathbf{a}_{1}, \ldots, \mathbf{a}_{n}\right)\right) .
$$

It follows from the functoriality of $T$ and properties of $\omega$ (cf. Definition A.2) that $\theta_{n}$ is a well-defined map. This fact and all necessary coherence properties for the definition of an operad action are proven exactly as in May77, 1.5-1.7]. We just have to prove that $\theta_{n}$ is an equivariant map.

Since $W$ is an $\mathbb{R}[G]$-submodule of $\mathcal{U}$, it follows from Definition A.2(iii) that the map $j_{W}$ is $G$-equivariant. Also, from Definition A.2(iv) one knows that $\omega$ : 
$T V_{1} \times \cdots \times T V_{n} \rightarrow T\left(V_{1} \oplus \cdots \oplus V_{n}\right)$ is also equivariant. Therefore, given $g \in G$ one has:

$$
\begin{aligned}
g * \theta_{n}\left(f ; \sigma_{1}, \ldots, \sigma_{n}\right) & =g *\left(j_{W} \circ T(\widehat{f}) \circ \omega\left(\mathbf{a}_{1}, \ldots, \mathbf{a}_{n}\right)\right) \\
& =j_{W}\left\{g *\left[T(\widehat{f})\left(g^{-1} *\left(g *\left(\omega\left(\mathbf{a}_{1}, \ldots, \mathbf{a}_{n}\right)\right)\right)\right)\right]\right\} \\
& =j_{W} \circ T(g * \widehat{f}) \circ \omega\left(g * \mathbf{a}_{1}, \ldots, g * \mathbf{a}_{n}\right) \\
& :=\theta_{n}\left(g * f ; g * \sigma_{1}, \ldots, g * \sigma_{n}\right) .
\end{aligned}
$$

This concludes the proof.

Definition A.6. Given an action of $\mathcal{L}_{*}(\mathcal{U})$ on a $G$-space $X$, the map $\mathcal{L}_{2} \times X \times X \rightarrow$ $X$ gives $X$ the structure of an equivariant $H$-space. In this case, we say that $X$ is $G$-group complete if for every subgroup $N \leq G$ the fixed point space $X^{N}$ is a group-like $H$-space in the usual sense; in other words, $\pi_{0}\left(X^{N}\right)$ becomes a group under the operation induced by the $H$-space structure.

The recognition principle, proven in CW91, is the following theorem. See Definition 1.1

Theorem A.7. Let $\mathcal{C}$ be a $C W E_{\infty} G$-operad, and let $X$ be a group-like based $G$-space of the homotopy type of a $G$-CW-complex on which $\mathcal{C}$ acts. Then $X$ is $G$-homotopy equivalent to an equivariant infinite loop space.

A.1. The case $G=\mathbb{Z} / 2$. When $G=\mathbb{Z} / 2$, our preferred complete $\mathbb{Z} / 2$-universe is $\left(\mathbb{C}^{\infty}, \sigma,\langle\cdot, \cdot\rangle\right)$, where $\sigma$ is the complex conjugation action on $\mathbb{C}^{\infty}$ and $\langle\cdot, \cdot\rangle$ is the usual Hermitian product in $\mathbb{C}^{\infty}$.

In this case, we can make a minor modification of the definition of a $\mathbb{Z} / 2 \mathcal{J}_{*}$-functor which is more suited to our constructions. Define $\mathbb{R J}_{*}$ as the category whose objects are the finite dimensional Real vector spaces $(V, \sigma)$ and whose morphisms between $(V, \sigma)$ and $(W, \tau)$ consist of the complex linear embeddings $j: V \rightarrow W$ between the underlying complex vector spaces. Note that $\operatorname{Mor}_{\mathbb{R} J_{*}}((V, \sigma),(W, \tau))$ becomes a Real space in the usual fashion, and hence the category $\mathbb{R} \mathcal{J}_{*}$ in enriched over $\mathbb{Z} / 2 \mathcal{T}$.

Definition A.8. A $\mathbb{R J}_{*}$-functor is a continuous covariant functor $T: \mathbb{R} \mathcal{J}_{*} \rightarrow \mathcal{T}_{\mathbb{Z} / 2}$ together with a (coherently) commutative, associative and continuous natural transformation

$$
\omega: T \times T \rightarrow T \circ \oplus .
$$

Writing $T V$ to denote $T(V, \sigma)$, we require that $T$ satisfies conditions (i) through (iv) in Definition A.2, with $G$ replaced by $\mathbb{Z} / 2$ and $G J_{*}$ replaced by $\mathbb{R J}_{*}$.

Let $\mathcal{F}$ denote the directed system consisting of the finite dimensional Real subspaces $(V, \sigma)$ of $\left(\mathbb{C}^{\infty}, \sigma\right)$ and their inclusions. Given an $\mathbb{R} J_{*}$-functor $T$, define

$$
T\left(\mathbb{C}^{\infty}\right):=\underset{(V, \sigma) \in \mathcal{F}}{\lim _{\vec{F}}} T V .
$$

Proposition A.9. Given an $\mathbb{R J}_{*}$-functor $T, T\left(\mathbb{C}^{\infty}\right)$ has a natural action of the equivariant linear isometries operad $\mathcal{L}_{*}\left(\mathbb{C}^{\infty}\right)$.

Proof. The proof is exactly the same as the proof of Proposition A.5, with $G$ replaced by $\mathbb{Z} / 2$. 


\section{ACKNOWLEDGEMENT}

The authors would like to thank H. Blaine Lawson, Jr., for fruitful conversations during the elaboration of this work. The first author thanks Texas A\&M University and the second author thanks the Instituto Técnico Superior (Lisbon) for their respective hospitality during the elaboration of this work.

\section{REFERENCES}

[Ati66] M. F. Atiyah, K-theory and reality, Quart. J. Math. Oxford 17 (1966), 367-386. MR 34:6756

[BLLF93] C. Boyer, H. B. Lawson, Jr., P. C. Lima-Filho, B. Mann, and M.-L. Michelsohn, Algebraic cycles and infinite loop spaces, Inventiones Math. 113 (1993), 373-388. MR 95a:55021

[Bot59] R. Bott, The stable homotopy of the classical groups, Ann. of Math. 70 (1959), 313337. MR 22:987

[CW91] S.R. Costenoble and S. Waner, Fixed set systems of equivariant infinite loop spaces, Trans. Amer. Math. Soc. 326 (1991), no. 2, 485-505. MR 91k:55015

[CW92] Equivariant Poincaré duality, Michigan Math. J. 39 (1992), no. 2, 325-351. MR 93d:55007

[dS03a] Pedro F. dos Santos, Algebraic cycles on real varieties and $\mathbb{Z} / 2$-homotopy theory, Proc. London Math. Soc. 86 (2003), 513-544. MR 2004c:55026

[dS03b] A note on the equivariant Dold-Thom theorem, J. Pure and Appl. Algebra 183 (2003), 299-312. MR 2004b:55021

[Dug01] D. Dugger, An Atiyah-Hirzebruch spectral sequence for KR-theory, Preprint, 2001.

[Dup69] Johan L. Dupont, Symplectic bundles and KR-theory, Math. Scand. 24 (1969), 27-30. MR 40:8046

[Dup99] , A note on characteristic classes for Real vector bundles, Preprint, 1999.

[FL92] Eric Friedlander and H. B. Lawson, Jr., A theory of algebraic cocycles, Ann. of Math. 136 (1992), no. 2, 361-428. MR 93g:14013

[Gro57] Alexander Grothendieck, Sur quelques points d'algèbre homologique, Tôhoku Math. J. 9 (1957), no. 2, 119-221. MR 21:1328

[Kah87] B. Kahn, Construction de classes de Chern équivariantes pour un fibré vectoriel réel, Comm. in Algebra 15 (1987), no. 4, 695-711. MR 88f:57042

[Lam90] T. K. Lam, Spaces of real algebraic cycles and homotopy theory, Ph.D. thesis, SUNY at Stony Brook, 1990.

[Law89] H. B. Lawson, Jr., Algebraic cycles and homotopy theory, Ann. of Math. 129 (1989), no. 2, 253-291. MR 90h:14008

[LF94] Paulo Lima-Filho, The topological group structure of algebraic cycles, Duke Math. J. 75 (1994), no. 2, 467-491. MR 95i:14010

[LF97] On the equivariant homotopy of free abelian groups on $g$-spaces and $g$-spectra, Math. Z. 224 (1997), 567-601. MR 98i:55014

[LLFM96] H. B. Lawson, Jr., P. C. Lima-Filho, and M.-L. Michelsohn, Algebraic cycles and equivariant cohomology theories, Proc. London Math. Soc. 73 (1996), no. 3, 679-720. MR 97i:55022

[LLFM98a] — On equivariant algebraic suspension, J. Alg. Geometry 7 (1998), 627-650. MR 99m:14016

[LLFM98b] _ Spaces of algebraic cycles and classical groups, Part I: Real cycles, Topology 42 (2003), 467-506. MR 2003m:14013

[LLFM98c] - Spaces of algebraic cycles and classical groups, Part II: Quaternionic cycles, Preprint, 1998.

[LM88] H. B. Lawson, Jr. and M. L. Michelsohn, Algebraic cycles, Bott periodicity, and the Chern characteristic map, Proc. of Symp. in Pure Math. 48 (1988), 241-264. MR 90d:14010

[LM91] Algebraic cycles and group actions, Differential Geometry, Longman Press, 1991, pp. 261-278. MR 93g:14007

[May77] J. P. May, $e_{\infty}$-ring spaces and $e_{\infty}$-ring spectra, Lecture Notes in Mathematics, vol. 577, Springer-Verlag, 1977. MR 58:13008 
[May72] J. P. May, The geometry of iterated loop spaces, Lecture Notes in Mathematics, vol. 271, Springer-Verlag, 1972. MR 54:8623b

[May86] L. G. Lewis, Jr., J. P. May, M. Steinberger, and J. E. McClure, Equivariant stable homotopy theory, Lecture Notes in Mathematics, vol. 1213, Springer-Verlag, 1986. MR 88e:55002

[May96] Equivariant homotopy and cohomology theory, CBMS, vol. 91, American Mathematical Society, 1996. MR 97k:55016

[Mos98] J. Mostovoy, Algebraic cycles and anti-holomorphic involutions on projective spaces, Bol. Soc. Mat. Mexicana (3) 6 (2000), no. 2, 151-170. MR 2002a:14009

[Qui73] D. Quillen, Higher algebraci k-theory, Algebraic $K$-theory I (New York), Lecture Notes in Mathematics, vol. 341, Springer-Verlag, 1973, pp. 85-147. MR 49:2895

[Seg68] G. Segal, Equivariant k-theory, Inst. Hautes Études Sci. Publ. Math. 34 (1968), 129151. MR 38:2769

[Sey73] R. M. Seymour, The real K-theory of Lie groups and homogeneous spaces, Quart. J. Math. Oxford 24 (1973), 7-30. MR 47:7738

Departamento de Matemática, Instituto Superior Técnico, Lisbon, Portugal

E-mail address: pedfs@math.ist.utl.pt

Department of Mathematics, Texas A\&M University, College Station, Texas 77843

E-mail address: plfilho@math.tamu.edu 